Review Article

\title{
Electroacupuncture for Gastrointestinal Function Recovery after Gynecological Surgery: A Systematic Review and Meta-Analysis
}

\author{
Xiang Gao, ${ }^{1}$ Yuzhuo Zhang, ${ }^{2}$ Yizhe Zhang, ${ }^{1}$ YuTzu Ku, ${ }^{2}$ and Yi Guo ${ }^{2}$ \\ ${ }^{1}$ Shanxi Province Hospital of Traditional Chinese Medicine, Shanxi Provincial Institute of Traditional Chinese Medicine, \\ Taiyuan 030012, China \\ ${ }^{2}$ Guangzhou University of Chinese Medicine, Guangzhou 510120, China
}

Correspondence should be addressed to Yi Guo; doctorguo1010@163.com

Received 13 July 2021; Revised 3 September 2021; Accepted 6 November 2021; Published 21 December 2021

Academic Editor: Ying Li

Copyright (C) 2021 Xiang Gao et al. This is an open access article distributed under the Creative Commons Attribution License, which permits unrestricted use, distribution, and reproduction in any medium, provided the original work is properly cited.

Background. Evidence for the efficacy and safety of electroacupuncture (EA) on gastrointestinal function recovery after gynecological surgery is unclear. Objective. This meta-analysis aimed to evaluate the effects of EA on recovery of postoperative gastrointestinal function for patients receiving gynecological surgery. Data sources: PubMed, Cochrane Central Register of Controlled Trials (CINAHL), Embase, China National Knowledge Infrastructure (CNKI), Weipu (CQVIP), and Wanfang databases were systematically searched from the inception dates to May 30, 2020, for relevant randomized controlled trials (RCTs). Study selection: RCTs that evaluated EA for postoperative gastrointestinal function directly related to gynecological surgery in adults aged 18 years or over. Data extraction and synthesis: paired reviewer independently extracted the data and assessed study quality. Standardized mean differences (SMD) were calculated as the effect measure from a random effects model. Main outcomes and measures: time to first flatus (TFF), time to bowel sounds recovery (TBS), and time to first defecation (TFD) were recorded as primary outcomes; postoperative nausea and vomiting (PONV), motilin (MTL), gastrin (GAS), $\mathrm{pH}$ value of gastric mucosa (pHi), gastric mucosal partial pressure of carbon dioxide (PgCO2), vasoactive intestinal peptide (VIP), and adverse event were reported as secondary outcomes. Results. We included eighteen RCTs (1117 participants). Our findings suggested that compared to the control group (CG), electroacupuncture group (EG) showed significant effects on TFF (SMD $=-0.98,95 \%$ CI: $[-1.28,-0.68], P<0.00001, I^{2}=69 \%$ ), TBS (SMD $=-0.98,95 \%$ CI: $[-1.84,-0.12], P=0.03, I^{2}=92 \%$ ), and TFD (SMD $\left.=-1.23,95 \% \mathrm{CI}:[-1.59,-0.88], P<0.0001, I^{2}=0 \%\right)$. Moreover, the incidence of PONV at postoperative $6 \mathrm{~h}(\mathrm{OR}=0.42$, 95\% CI: [0.27, 0.64], $\left.P<0.0001, I^{2}=0 \%\right)$ and $24 \mathrm{~h}\left(\mathrm{OR}=0.46,95 \% \mathrm{CI}:[0.32,0.68], P<0.0001, I^{2}=0 \%\right)$ was lower in the EG than that in the CG, whereas no significant difference in ratio of PONV at postoperative $48 \mathrm{~h}\left(\mathrm{OR}=0.55,95 \% \mathrm{CI}:[0.20,1.51], P=0.25, I^{2}=0 \%\right)$ was detected between the two groups. Meanwhile, there was a significant effect in favor of EA on the level of MTL at postoperative $6 \mathrm{~h}$ $\left(\mathrm{SMD}=-0.93,95 \% \mathrm{CI}:[-1.36,-0.61], P<0.0001, I^{2}=21 \%\right)$, while no significant effect was observed at postoperative $24 \mathrm{~h}(\mathrm{SMD}=-0.43$, 95\% CI: $\left.[-0.89,0.02], P=0.06, I^{2}=69 \%\right)$ in the EG when compared to the CG. Additionally, a large significant effect on decreasing $\mathrm{PgCO}_{2}$ was found in the EG in comparison to the CG, but no significant effect in favor of EA on GAS, VIP, or pHi was observed. It was reported that there was one participant with pain at the needling sites and bruising, and three participants withdrew because they were not intolerant to EA. Conclusions. EA could be a promising strategy for the prevention and treatment of gastrointestinal dysfunction after gynecological surgery, including shortening TFF and TFD, TBS, regulating MTL, and decreasing the ratio of PONV within postoperative 24h. The effects on MTL and PONV varied with different intervention points, and EA used at 30 min prior to surgery might be recommended. However, the evidence quality ranged from low to very low, and large-scale and high-quality RCTs were warranted.

\section{Introduction}

Postoperative recovery of gastrointestinal function was considered as one of the most important parts for the rehabilitation after surgery, which was a condition that mainly related to surgical stress, anesthesia regimen, surgical treatment, and postoperative analgesia method [1]. The short-term gastrointestinal dysfunction after surgery was referred to as postoperative ileus (POI), which could cause undesirable consequences, including abdominal distension, 
lack of flatus and defecation, and nausea and vomiting. As was reported, POI would influence postoperative experience, increase the length of hospital stay, and even raise the risk of morbidity and mortality, which not only strongly prevented rehabilitation after surgery, but also posed a substantial economic burden on family and society [2].

POI was frequently observed after gastroenterological surgery, and it was followed by gynecological surgery [3]. It was reported that the prevalence of POI ranged from 5\% to $25 \%$ among patients undergoing gynecological surgery [4], and even the incidence was up to $50 \%$ in patients receiving surgical therapy for gynecological cancers [5]. In recent years, minimally invasive surgery, such as laparoscopy surgery, has become more commonly used for gynecologic indications [6]. However, the incidence of gastrointestinal dysfunction following surgery is not decreased [7]. Even though increasing studies focusing on improving recovery of postoperative gastrointestinal function have been conducted, strategies with satisfactory efficacy are still rarely reported, especially for gynecological population. Thus, apart from improving anesthesia regimen and surgical technique, so far it is still urgent and essential to develop an effective and safety method of promoting the return of gastrointestinal function for patients receiving gynecological surgery.

Acupuncture, as one of the conventional Chinese medical therapies, has been applied to promote gastrointestinal function for thousands of years [8]. Electroacupuncture (EA) is a modified technique involving traditional acupuncture and electrical stimulation to achieve a greater response, which has been proved to be a promising approach to reduce complications and accelerate rehabilitation after surgery in the field of orthopedic, abdominal, and gynecological diseases. Moreover, there are an increasing number of studies focusing on EA for treatment and prevention of POI. But the results concerning the efficacy of EA on POI are inconsistent $[9,10]$. It was reported in the previous meta-analyses [11-14] that supported the benefits of EA/acupuncture to POI, but the efficacy of EA on POI for gynecological patients still lacked evidence basis. Gynecological surgery was unlike gastroenterological surgery or other surgery in terms of pathology and surgical methods, which might lead to difference outcomes of gastrointestinal function recovery after surgery. Consequently, the purpose of this study was to evaluate the safety and efficacy of EA on postoperative recovery of gastrointestinal function for the patients receiving gynecological surgery, which could provide new evidence for promoting rehabilitation after gynecological surgery.

\section{Methods}

This study was conducted according to the Preferred Reporting Items for Systematic Reviews and Meta-Analyses (PRISMA) guidelines [15]. Moreover, the protocol of this study was registered in PROSPERO, and the registration number is CRD42021260096. In the process of retrieval, we found that most of the literatures were published in Chinese, so we retrieved with increased numbers of Chinese database.
However, due to the delay of preliminary preparation time, the deadline for literature retrieval was extended to May 30, 2021. We had submitted the amendments in the registration system.

2.1. Search Strategy. In order to identify relevant studies, we systematically search the electronic databases including PubMed, Cochrane Central Register of Controlled Trials (CINAHL), Embase, China National Knowledge Infrastructure (CNKI), Weipu (CQVIP), and Wanfang from inception to May 30, 2021. The search terms, such as "postoperative ileus," "postoperative gastrointestinal motility disorder," "postoperative gastrointestinal function recovery," "postoperative gastrointestinal dysfunction," "postoperative gastrointestinal function," "postoperative nausea and vomiting," and "electroacupuncture," were used to search in each database without language or disease restrictions. The search strategy was described in Supplementary Figure 1.

2.2. Inclusion Criteria. We formulated inclusion and exclusion criteria based on PICOS (patients, intervention, comparator, outcomes, and study design) approach [16].

\subsubsection{Patients}

(i) Subjects aged 18 or over

(ii) Subjects with gynecological disease receiving surgical treatment

2.2.2. Interventions. EA should be used in the experimental group in the included study.

2.2.3. Comparators. EA versus (vs) other therapy, EA+ other therapy vs. other therapy, EA vs. nonintervention.

2.2.4. Outcomes. The literature reporting more than or equal to one index for assessing gastrointestinal function after surgery would be included in this study. The outcomes were listed as follows:

\section{Primary Outcomes}

(i) Time to first flatus (TFF)

(ii) Time to bowel sounds recovery (TBS)

(iii) Time to first defecation (TFD) Secondary Outcomes

(i) Postoperative nausea and vomiting (PONV)

(ii) Motilin (MTL)

(iii) Gastrin (GAS)

(iv) $\mathrm{pH}$ value of gastric mucosa $(\mathrm{pHi})$

(v) Gastric mucosal partial pressure of carbon dioxide $\left(\mathrm{PgCO}_{2}\right)$

(vi) Vasoactive intestinal peptide (VIP)

(vii) Adverse event 


\subsubsection{Study Design}

(i) Clinical randomized controlled trials (RCTs)

(ii) Published in a peer-reviewed journal

(iii) Language: Chinese or English

2.3. Exclusion Criteria. (1) Pregnancy, or participants receiving obstetric surgery; (2) manual acupuncture, acupoint massage, transcutaneous electrical nerve stimulation, transcutaneous electrical acupoints stimulation, and neuromuscular electrical stimulation; (3) repeated publications, conference abstracts, comments, protocol, meta-analysis, or reviews, or full-text unavailable articles.

2.4. Literature Screening. Paired investigators (X. Gao and Yu Z. Zhang) independently screened the retrieved studies. Firstly, all the studies were imported to EndNote X8 (Bld 10063) to remove duplicates. Secondly, the remaining studies were preliminarily selected by reading the titles and abstracts. Thirdly, the included studies were identified based on the inclusion and exclusion criteria by full-text reading.

2.5. Data Extraction. The relevant data were independently extracted from the included studies by the two reviewers, including study characteristics (e.g., author names, publication year, study design, and sample size), participant characteristics (e.g., age, year), intervention type, intervention characteristics (e.g., acupoints, model, and intensity), and outcomes. During the process of screening and data extraction, any discrepancies would be resolved by discussion, or consultation with a third reviewer (Y. Guo) until a consensus was reached.

2.6. Quality Assessment. In accordance with the Cochrane Collaboration's Risk of Bias tool V 2.0, the two reviewers assessed the quality of the included literatures from seven dimensions: bias arising from the randomisation process, bias from deviations from the intended interventions, bias from missing outcome data, bias due to measurement of the outcome, bias from the selection of the reported results, and overall risk of bias [16]. The risk of each item is divided into three levels: high, unclear, and low. Meanwhile, the two researchers evaluated the methodological quality of included studies according to the Physiotherapy Evidence Database (PEDro) scale [17], which includes 10 items for assessment of trial quality from various aspects, including randomisation procedure, concealed allocation, similar baseline, patients blinding, therapists blinding, assessors blinding, adequate follow-up (dropout rate $<15 \%$ ), intention to treat analysis, between-group statistical analysis, and point and variability measures; the total score ranged from 0 to 10 . According to the total score, quality of study was categorized into three degrees, including high $(10 \geq$ PEDro score $\geq 6$ ), fair $(6>$ PEDro score $\geq 4)$, and low $(0 \leq$ PEDro score $\leq 3)$ [18].

2.7. Statistical Analysis. We conducted the data analysis by using review manager (version 5.3, the Nordic Cochrane Centre, Copenhagen, Denmark) and Stata (version 13.0, the
StataCorp LP, TX, USA). For continuous variables, standardized mean differences (SMDs) and 95\% confidence intervals $(95 \% \mathrm{CI})$ were calculated as the effect measure by using a random effects model, and standard mean effect sizes were divided to different categories, including no change (0), small effect (0.2), moderate effect (0.5), and large effect (0.8) [18]. Enumeration data was analyzed by using a random effects method and expressed as the odds ratios (OR) and 95\% CI. All the corresponding meta-analysis results were illustrated by the forest map intuitively. Cochran's Q-test and $I^{2}$ index were employed to estimate heterogeneity [19], and $I^{2}$ statistic greater than $50 \%$ was considered as substantially heterogeneous. When a substantially heterogeneous was observed, subgroups analysis or sensitivity analyses were used to determine the risk factor resulted in the high heterogeneity. Grading of recommendations, assessment, development, and evaluation (GRADE) approach was utilized to evaluate the quality of evidence [20]. In addition, publication bias was assessed by Begg's and Egger's tests [19], and the visual inspection of funnel plots would be applied if the index was reported in more than 10 included studies. The significant difference level was set at $P<0.05$.

\section{Results}

3.1. Study Selection. A total of 2889 potentially relevant strings were retrieved from the Chinese and English databases. After removing duplicates and screening titles and reading summary, 2857 trials were eliminated. Consequently, the remaining 32 studies were screened by reading full text. Finally, eighteen RCTs [21-38] fulfilled the inclusion criteria, and 1117 subjects including 557 subjects in the electroacupuncture group (EG) and 560 subjects in the control group (CG) were involved. Flowchart of the structured review is illustrated in Figure 1.

3.2. Study Characteristics. The included studies involved 1117 subjects who received gynecological surgery. Seven studies $[22,23,25-27,32,33]$ reported the effects of EA on postoperative gastrointestinal function recovery for patients undergoing total abdominal hysterectomy, and eleven studies [21, 24, 28-31, 34-38] involved laparoscopic surgery. Anesthesia type included general anesthesia and epidural anesthesia $[25,33]$. In addition, EA was performed at $24 \mathrm{~h}$ prior to surgery in three studies $[16,21,30]$, at $30 \mathrm{~min}$ prior to surgery or before the start of surgery in ten studies [22, 24, 28, 29, 31, 34-38], and after surgery in five studies $[23,25,27,32,33]$. Among all the acupoints involved in the included studies, Zusanli (ST36) (14/ 18) and Neiguan (PC6) (11/15) were most frequently selected, while less frequently selected acupoints included Hegu (LI4) (4/ 18), Shangjuxu (ST37) (3/18), Zhongwan (RN12) (3/18), Tianshu (ST25) (3/18), Liangqiu (ST34) (2/18), Sanjinjiao (SP6) (2/18), Liangmen (ST21) (2/18), Xuehai (SP10) (1/18), and Taichong (LR3) (1/18). Tables 1 and 2 presented the characteristics of each included study.

3.3. Risk of Bias in Included Studies. The detailed results are depicted in Figure 2. All the included studies were reported as random generation, and four of the RCTs $[21,22,29,38]$ were 


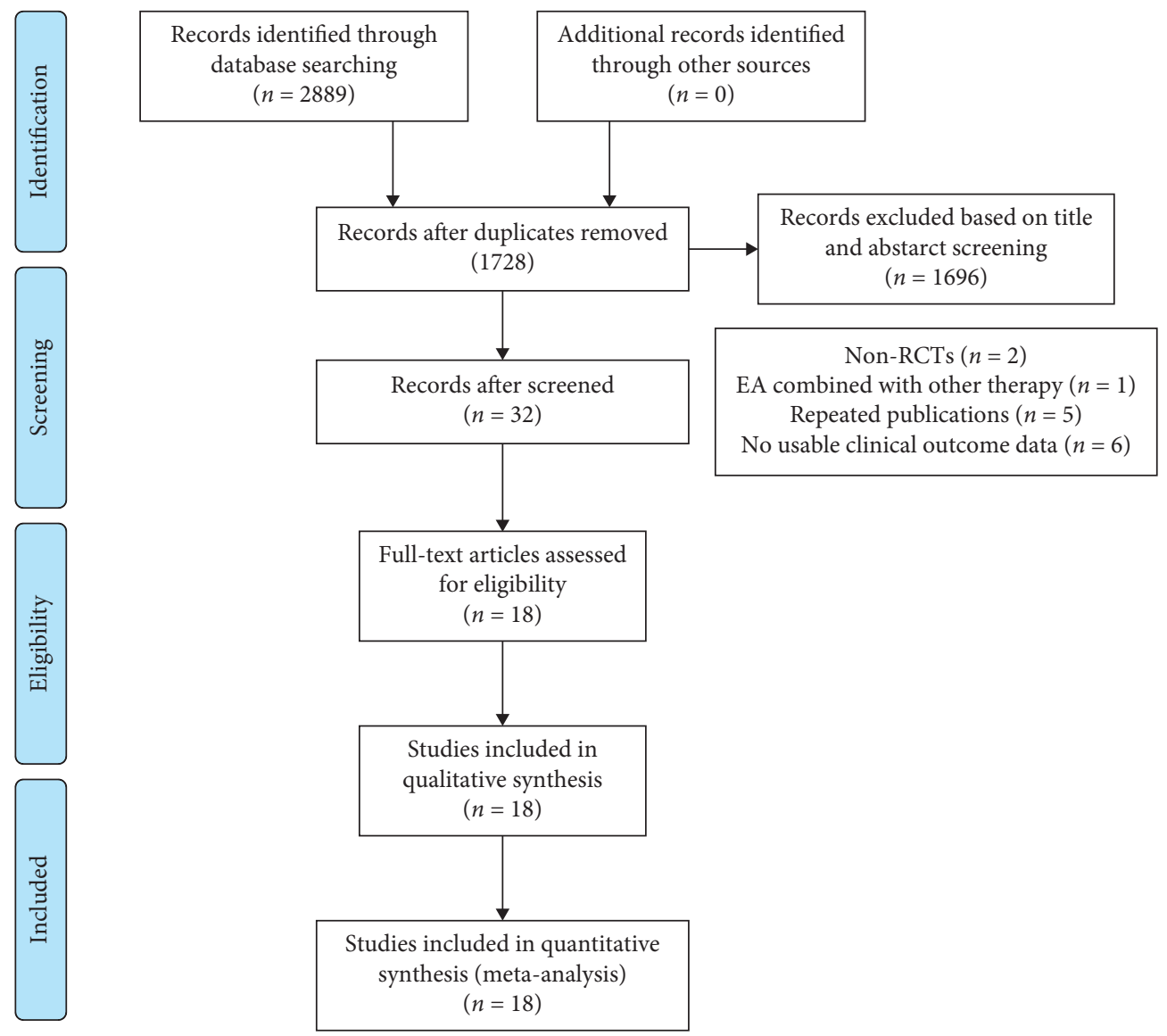

FIgURE 1: Flowchart of study selection.

conducted with concealed allocation. Participants were blinded to the group allocation in four studies [21, 22, 29, 38], and assessment blinding was reported in seven RCTs $[21,22,24,27,29,36,38]$. Meanwhile, based on the evaluation of PEDro, all the scores of the included studies ranged from 5 to 9 (mean score + standard deviation $=6.61 \pm 1.42$; Supplementary Table 1), which indicated that the methodological quality of included studies was fair to high. All the included studies were described as random generation, while blinding was recorded only in four trials $[21,22,29,38]$. Additionally, none of the studies reported a follow-up rate of more than $15 \%$, and intention-to-treat analysis was not found in any of the included studies.

\subsection{Meta-Analysis}

\subsubsection{Primary Outcomes}

(1) Time to First Flatus. Ten of the included studies reported TFF $[21,23,25,26,28-30,32-34]$, and result from metaanalysis suggested that, overall, EG showed a significantly effect on TFF compared to the CG (SMD $=-0.98,95 \%$ CI: $[-1.28$, $\left.-0.68], P<0.00001, I^{2}=69 \%\right)$. Moreover, TFF was significantly shorter in the EG than that in the CG, either for laparoscopic surgery $(\mathrm{SMD}=-0.97,95 \% \mathrm{CI}$ : $[-1.23,-0.72], P<0.00001$, $\left.I^{2}=0 \%\right)$ or total abdominal hysterectomy (SMD $=-1.01,95 \%$
CI: [-1.57, -0.44$], P=0.0005, I^{2}=84 \%$ ) (Figure 3). Furthermore, we conducted the subgroup analysis by anesthesia types, intervention points, and comparators. All the results supported that EA shortened TFF, no matter for general anesthesia $\left(\mathrm{SMD}=-1.01,95 \% \mathrm{CI}:[-1.37,-0.65], P<0.00001, I^{2}=70 \%\right)$ or epidural anesthesia (SMD $=-0.88,95 \% \mathrm{CI}$ : $[-1.61,-0.16]$, $P=0.02, I^{2}=81 \%$ ) (Supplementary Figure 2), EA applied at $24 \mathrm{~h}$ prior to surgery $(\mathrm{SMD}=-1.23,95 \% \mathrm{CI}$ : $[-1.73,-0.73]$, $\left.P<0.00001, I^{2}=51 \%\right), 30 \mathrm{~min}$ prior to surgery $(\mathrm{SMD}=-0.97$, 95\% CI: $\left.[-1.37,-0.57], P<0.00001, I^{2}=36 \%\right)$, or after surgery $\left(\mathrm{SMD}=-0.84,95 \% \mathrm{CI}:[-1.41,-0.26], P=0.004, I^{2}=83 \%\right)$ (Supplementary Figure 3). And a shorter TFF was observed in the EG than that in the CG by comparators as EA VS control $\left(\mathrm{SMD}=-1.27,95 \% \mathrm{CI}:[-1.52,-1.03], P<0.00001, I^{2}=0 \%\right)$, $\mathrm{EA}+$ other therapy vs. other therapy $(\mathrm{SMD}=-1.40,95 \% \mathrm{CI}$ : $\left.[-2.03,-0.77], P<0.0001, I^{2}=84 \%\right)$, and EA vs. ginger partitioned moxibustion on umbilicus ( $\mathrm{SMD}=-0.35,95 \% \mathrm{CI}$ : $[-0.67,-0.03], P=0.03, I^{2}=1 \%$ ) (shown in Supplementary Figure 4). However, there was no significant difference in the ratio of subjects with $\mathrm{TFF}>72 \mathrm{~h}(\mathrm{OR}=0.16,95 \% \mathrm{CI}$ : [0.02, 1.35], $P=0.09, I^{2}=0 \%$ ) (Figure 4 ).

(2) Time to Bowel Sounds Recovery. Meta-analysis of four studies showed a significantly shorter TBS for the EG compared to the CG (SMD $=-0.98,95 \%$ CI: $[-1.84,-0.12]$, $\left.P=0.03, I^{2}=92 \%\right)$. Nevertheless, subgroup analysis showed that EG presented a shorter TBS when EA performed at 
TABle 1: Characteristics of the included studies.

\begin{tabular}{|c|c|c|c|c|c|c|c|}
\hline \multirow[t]{2}{*}{ First author (year) } & \multicolumn{2}{|c|}{ Age (years) } & \multirow{2}{*}{$\begin{array}{c}\text { Sample } \\
\text { size } \\
\text { EG/CG }\end{array}$} & \multirow[t]{2}{*}{ Type of surgery } & \multirow[t]{2}{*}{ Type of anesthesia } & \multicolumn{2}{|c|}{$\begin{array}{c}\text { Duration of anesthesia/ } \\
\text { surgery }\end{array}$} \\
\hline & EG & CG & & & & EG & CG \\
\hline Li et al. (2017) [21] & $35.2 \pm 6.1$ & $34.4 \pm 9.1$ & $20 / 20$ & Laparoscopic surgery & $\begin{array}{c}\text { General anesthesia } \\
\text { (I/II) }\end{array}$ & $89.3 \pm 38.9$ & $95.5 \pm 32.8$ \\
\hline $\begin{array}{l}\text { Praveena et al. } \\
(2016)[22]\end{array}$ & $47.5 \pm 7.94$ & $48.72 \pm 6.72$ & $32 / 32$ & $\begin{array}{l}\text { Total abdominal } \\
\text { hysterectomy }\end{array}$ & $\begin{array}{c}\text { General anesthesia } \\
(\mathrm{I} / \mathrm{II})\end{array}$ & $149.06 \pm 42.64$ & $151.97 \pm 50.71$ \\
\hline Bai et al. (2012) [23] & \multicolumn{2}{|c|}{$42 \sim 60$} & $30 / 30$ & $\begin{array}{c}\text { Total abdominal } \\
\text { hysterectomy }\end{array}$ & $\begin{array}{l}\text { General anesthesia } \\
\text { (I/II) }\end{array}$ & - & - \\
\hline $\begin{array}{l}\text { Chen et al. (2014) } \\
\text { [24] }\end{array}$ & \multicolumn{2}{|c|}{$18 \sim 65$} & $27 / 27$ & Laparoscopic surgery & $\begin{array}{c}\text { General anesthesia } \\
(\mathrm{I} / \mathrm{II})\end{array}$ & $95.2 \pm 39.4$ & $77.8 \pm 23.8$ \\
\hline $\begin{array}{l}\text { Jin and Jing (2015) } \\
\text { [25] }\end{array}$ & \multicolumn{2}{|c|}{$35 \sim 66(48.3 \pm 6.3)$} & $45 / 45$ & $\begin{array}{l}\text { Total abdominal } \\
\text { hysterectomy }\end{array}$ & $\begin{array}{c}\text { Epidural } \\
\text { anesthesia }\end{array}$ & - & - \\
\hline Li et al. (2019) [26] & $37.26 \pm 8.83$ & $36.96 \pm 9.05$ & $30 / 30$ & $\begin{array}{c}\text { Total abdominal } \\
\text { hysterectomy }\end{array}$ & $\begin{array}{c}\text { General anesthesia } \\
\text { (I/II) }\end{array}$ & - & - \\
\hline Lu et al. (2010) [27] & \multicolumn{2}{|c|}{$40 \sim 60$} & $29 / 30$ & $\begin{array}{c}\text { Total abdominal } \\
\text { hysterectomy }\end{array}$ & $\begin{array}{c}\text { General anesthesia } \\
(\mathrm{I} / \mathrm{II})\end{array}$ & - & - \\
\hline Toronui (2019) [28] & $40.54 \pm 5.82$ & $40.62 \pm 5.84$ & $29 / 29$ & Laparoscopic surgery & General anesthesia & $84.31 \pm 35.21$ & $84.58 \pm 35.56$ \\
\hline $\begin{array}{l}\text { Wang et al. (2018) } \\
\text { [29] }\end{array}$ & $35.76 \pm 7.15$ & $34.88 \pm 7.28$ & $27 / 28$ & Laparoscopic surgery & $\begin{array}{c}\text { General anesthesia } \\
(\mathrm{I} / \mathrm{II})\end{array}$ & $68.69 \pm 29.34$ & $67.62 \pm 34.42$ \\
\hline Wang et al. (2018) & $34.9 \pm 5.1$ & $34.8 \pm 6.2$ & $27 / 28$ & Laparoscopic surgery & $\begin{array}{c}\text { General anesthesia } \\
\text { (I/II) }\end{array}$ & $80.2 \pm 36.9$ & $85.3 \pm 31.8$ \\
\hline Wang et al. (2011) & $41.2 \pm 6.1$ & $39.3 \pm 8.5$ & $30 / 30$ & Laparoscopic surgery & $\begin{array}{c}\text { General anesthesia } \\
(\mathrm{I} / \mathrm{II})\end{array}$ & $67.2 \pm 16.0$ & $70.0 \pm 10.2$ \\
\hline $\begin{array}{l}\text { Wang and Xi (2017) } \\
\text { [32] }\end{array}$ & $31.3 \pm 4.5$ & $31.5 \pm 4.3$ & $40 / 40$ & $\begin{array}{c}\text { Total abdominal } \\
\text { hysterectomy }\end{array}$ & $e^{-2}(2 \pi)$ & $138 \pm 24$ & $132 \pm 30$ \\
\hline $\begin{array}{l}\mathrm{Xi} \text { and Wang (2015) } \\
{[33]}\end{array}$ & $33 \pm 3$ & $33 \pm 3$ & $37 / 38$ & $\begin{array}{c}\text { Total abdominal } \\
\text { hysterectomy }\end{array}$ & $\begin{array}{l}\text { Epidural } \\
\text { anesthesia }\end{array}$ & $103.2 \pm 30.6$ & $102.6 \pm 31.8$ \\
\hline $\begin{array}{l}\text { Yang et al. (2012) } \\
{[34]}\end{array}$ & $32 \pm 9$ & $30 \pm 10$ & $30 / 30$ & Laparoscopic surgery & $\begin{array}{c}\text { General anesthesia } \\
(\mathrm{I} / \mathrm{II})\end{array}$ & $78 \pm 23$ & $80 \pm 25$ \\
\hline $\begin{array}{l}\text { Huang et al. (2021) } \\
\text { [35] }\end{array}$ & 31 - & -56 & $30 / 30$ & Laparoscopic surgery & $\begin{array}{c}\text { General anesthesia } \\
(\mathrm{I} / \mathrm{II})\end{array}$ & - & - \\
\hline $\begin{array}{l}\text { Ye and Huang } \\
(2019)[36]\end{array}$ & $33 \pm 9$ & $35 \pm 10$ & $40 / 40$ & Laparoscopic surgery & $\begin{array}{c}\text { General anesthesia } \\
(\mathrm{I} / \mathrm{II})\end{array}$ & $97 \pm 9$ & $95 \pm 10$ \\
\hline $\begin{array}{l}\text { Yu and Ning (2016) } \\
\text { [37] }\end{array}$ & $31 \pm 4.4$ & $30 \pm 5.4$ & $27 / 26$ & Laparoscopic surgery & $\begin{array}{c}\text { General anesthesia } \\
(\mathrm{I} / \mathrm{II})\end{array}$ & $78.1 \pm 16.2$ & $75.0 \pm 13.7$ \\
\hline $\begin{array}{l}\text { Zhang et al. (2013) } \\
\text { [38] }\end{array}$ & $34.74 \pm 5.64$ & $36.81 \pm 9.26$ & $27 / 27$ & Laparoscopic surgery & $\begin{array}{c}\text { General anesthesia } \\
\text { (I/II) }\end{array}$ & - & - \\
\hline
\end{tabular}

$30 \mathrm{~min}$ prior to surgery $(\mathrm{SMD}=-2.14,95 \% \mathrm{CI}$ : $[-2.79$, -1.48], $P<0.00001, I^{2}=$ not applicable), whereas subjects receiving EA therapy after surgery in the EG showed no difference to the CG with respect to TBS (SMD $=-0.62,95 \%$ CI: $[-1.41,0.17], P=0.12, I^{2}=89 \%$ ) (Figure 5). In addition, there was no significant difference in TBS between patients receiving $\mathrm{EA}$ and ginger partitioned moxibustion on umbilicus (SMD $=-0.22$, 95\% CI: $[-0.53,-0.10], P=0.18$, $I^{2}=0 \%$ ) (Supplementary Figure 5).

(3) Time to First Defecation. In this study, two trials recorded TFD. Meta-analysis result revealed that, compared to the CG, TFD was significantly shorter in the EG $(\mathrm{SMD}=-1.23$, 95\% CI: $[-1.59,-0.88], P<0.0001, I^{2}=0 \%$ ) (Figure 6).

\subsubsection{Secondary Outcomes}

(1) Postoperative Nausea and Vomiting (PONV). The incidence of PONV at postoperative $6 \mathrm{~h}, 24 \mathrm{~h}$, and $48 \mathrm{~h}$ was reported in eight $[21-23,27,29-31,35]$, ten [21-23, 27, 29-31, 35-37], and four studies [23, 27, 29, 31], respectively. Meta-analysis results suggested a lower incidence of PONV at postoperative $6 \mathrm{~h}(\mathrm{OR}=0.42,95 \% \mathrm{CI}$ : $\left.[0.27,0.64], P<0.0001, I^{2}=0 \%\right), 24$ h $(\mathrm{OR}=0.46,95 \% \mathrm{CI}$ : $\left.[0.32,0.68], P<0.0001, I^{2}=0 \%\right)$ in the EG than that in the $\mathrm{CG}$, whereas no significant difference in ratio of PONV at postoperative $48 \mathrm{~h}(\mathrm{OR}=0.55,95 \% \mathrm{CI}$ : $[0.20,1.51], P=0.25$, $I^{2}=0 \%$ ) was detected between the two groups (Figure 7). Subgroup analysis revealed that no difference in the ratio of PONV at postoperative $6 \mathrm{~h}$ was observed between the two groups when EA performed at $24 \mathrm{~h}$ prior to surgery (OR $=0.53$, 95\% CI: $\left.[0.22,1.27], P=0.15, I^{2}=0 \%\right)$ or after surgery $\left(\mathrm{OR}=0.48,95 \% \mathrm{CI}\right.$ : $\left.[0.22,1.06], P=0.07, I^{2}=0 \%\right)$, but its ratio was lower in the EG when EA was performed at $30 \mathrm{~min}$ prior to surgery in comparison to the $\mathrm{CG}(\mathrm{OR}=0.34$, 95\% CI: $\left.[0.19,0.63], P=0.0006, I^{2}=0 \%\right)$. Regarding the ratio of PONV at postoperative $24 \mathrm{~h}$, it was lower in the EG than that in the CG whenever EA was conducted at $24 \mathrm{~h}$ $\left(\mathrm{OR}=0.25,95 \% \mathrm{CI}:[0.09,0.67], P=0.006, I^{2}=0 \%\right)$ or 
TABLE 2: Interventions, outcomes, and study design on the included studies.

\begin{tabular}{|c|c|c|c|c|c|}
\hline $\begin{array}{l}\text { First author } \\
\text { (year) }\end{array}$ & Intervention & Intervention parameters & Intervention dose & Main outcome & $\begin{array}{l}\text { Study } \\
\text { design }\end{array}$ \\
\hline $\begin{array}{l}\text { Li et al. } \\
(2017)[21]\end{array}$ & $\begin{array}{l}\mathrm{EG}: \mathrm{EA}+\text { routine treatment; } \mathrm{CG}: \\
\quad \text { routine treatment. }\end{array}$ & $\begin{array}{l}\text { Acupoints: bilateral Neiguan } \\
\text { (PC6) and Zusanli (ST36), mode: } \\
\text { dense-disperse wave; frequency: } \\
\text { 20/100 Hz; intensity (mA) : } \\
\text { strong but comfortable. } 7548\end{array}$ & $\begin{array}{l}24 \text { hours prior to the } \\
\text { surgery, once for } \\
30 \text { min. }\end{array}$ & TFF, PONV & RCT \\
\hline $\begin{array}{l}\text { Praveena } \\
\text { et al. (2016) } \\
{[22]}\end{array}$ & $\begin{array}{l}\mathrm{EG}: \mathrm{EA}+\text { routine treatment, } \mathrm{CG}: \\
\text { routine treatment. }\end{array}$ & $\begin{array}{l}\text { acupoints: bilateral Hegu (LI4) } \\
\text { and Neiguan (PC6), mode: } \\
\text { continuous wave, frequency: } \\
2 \mathrm{~Hz} \text {; intensity (mA): Level } 1 \text {. }\end{array}$ & $\begin{array}{l}\text { Before the start of } \\
\text { surgery until the end } \\
\text { of surgery. }\end{array}$ & PONV & RCT \\
\hline $\begin{array}{l}\text { Bai et al. } \\
\text { (2012) [23] }\end{array}$ & $\begin{array}{c}\text { EG : EA + Tropisetron ( } 5 \mathrm{mg}, \\
\text { intravenous injection after anesthesia } \\
\text { induction) + routine treatment; CG : } \\
\text { Tropisetron ( } 5 \mathrm{mg} \text {, intravenous } \\
\text { injection after anesthesia } \\
\text { induction) + routine treatment. }\end{array}$ & $\begin{array}{l}\text { Acupoints: bilateral Neiguan } \\
\text { (PC6) and Zusanli (ST36), } \\
\text { Shangjuxu (ST37), Zhongwan } \\
\text { (RN12), Tianshu (ST25), mode: } \\
\text { unreported; frequency: } 2 \mathrm{~Hz} \text {; } \\
\text { intensity (mA): unclear. }\end{array}$ & $\begin{array}{l}\text { At hour } 5,23 \text {, and } 27 \\
\text { after surgery, } \\
30 \mathrm{~min} / \text { once. }\end{array}$ & $\begin{array}{l}\text { PONV, TFF, } \\
\text { MTL, GAS }\end{array}$ & RCT \\
\hline $\begin{array}{l}\text { Chen et al. } \\
\text { (2014) [24] }\end{array}$ & $\begin{array}{l}\mathrm{EG}: \mathrm{EA}+\text { routine treatment; CG: } \\
\text { routine treatment }\end{array}$ & $\begin{array}{l}\text { Acupoints: bilateral Liangqiu } \\
\text { (ST34) and Zusanli (ST36), } \\
\text { mode: continuous wave; } \\
\text { frequency: } 2 \mathrm{~Hz} \text {; intensity (mA): } \\
\text { maximum tolerable. }\end{array}$ & $\begin{array}{l}30 \text { minutes prior to } \\
\text { the surgery until the } \\
\text { end of surgery. }\end{array}$ & $\mathrm{PHi}, \mathrm{PgCO}_{2}$ & RCT \\
\hline $\begin{array}{l}\text { Jin and Jing } \\
(2015)[25]\end{array}$ & $\begin{array}{l}\mathrm{EG}: \mathrm{EA}+\text { routine treatment; } \mathrm{CG}: \\
\text { routine treatment }\end{array}$ & $\begin{array}{l}\text { Acupoints: bilateral Zusanli } \\
\text { (ST36); mode: unreported; } \\
\text { frequency and intensity (mA): } \\
\text { unclear. }\end{array}$ & $\begin{array}{l}\text { At hour } 5 \text { after } \\
\text { surgery, twice a day, } \\
\text { until time to first } \\
\text { flatus. }\end{array}$ & TBS, TFF, TFD & RCT \\
\hline $\begin{array}{l}\text { Li et al. } \\
(2019)[26]\end{array}$ & $\begin{array}{l}\mathrm{EG}: \mathrm{EA}+\text { routine treatment; CG: } \\
\text { routine treatment. }\end{array}$ & $\begin{array}{l}\text { Acupoints: bilateral Neiguan } \\
\text { (PC6), Xuehai (SP10), Hegu } \\
\text { (LI4) and Zusanli (ST36), mode: } \\
\text { dense-disperse wave; frequency: } \\
\text { 2/10/50/100 Hz; intensity (mA): } \\
\text { unclear. }\end{array}$ & $\begin{array}{l}24 \text { hours prior to the } \\
\text { surgery, once for } \\
30 \mathrm{~min} \text {. }\end{array}$ & TFF, PONV, & RCT \\
\hline $\begin{array}{l}\text { Lu et al. } \\
(2010)[27]\end{array}$ & $\begin{array}{l}\text { EG: EA + Tropisetron ( } 5 \mathrm{mg} \text {, } \\
\text { intravenous injection prior to the end } \\
\text { of surgery) + routine treatment; CG: } \\
\text { Tropisetron ( } 5 \mathrm{mg} \text {, intravenous } \\
\text { injection prior to the end of } \\
\text { surgery) + routine treatment. }\end{array}$ & $\begin{array}{l}\text { Acupoints: bilateral Neiguan } \\
\text { (PC6) and Zusanli (ST36), Hegu } \\
\text { (LI4), Sanyinjiao (SP6), Taichong } \\
\text { (LR3), mode: dense-disperse } \\
\text { wave; frequency: } 2 / 10 \mathrm{~Hz} \\
\text { intensity (mA): maximum } \\
\text { tolerable. }\end{array}$ & $\begin{array}{l}\text { At hours } 1,5 \text {, and } 23 \\
\text { after surgery, } \\
30 \text { min/once. }\end{array}$ & $\begin{array}{l}\text { PONV, } \\
\text { number of TFF } \\
\text { more than } 72 \mathrm{~h}\end{array}$ & RCT \\
\hline $\begin{array}{l}\text { Toronui } \\
(2020)[28]\end{array}$ & $\begin{array}{l}\mathrm{EG}: \mathrm{EA}+\text { routine treatment; CG: } \\
\text { routine treatment. }\end{array}$ & $\begin{array}{l}\text { Acupoints: bilateral Zusanli } \\
\text { (ST36), frequency: } 2 / 10 \mathrm{~Hz} .\end{array}$ & $\begin{array}{l}\text { Prior to the surgery, } \\
\text { once for } 15 \mathrm{~min} \text {. }\end{array}$ & $\begin{array}{l}\text { TBS, TFF, } \\
\text { TFD, PONV }\end{array}$ & RCT \\
\hline $\begin{array}{l}\text { Wang et al. } \\
(2018) \text { [29] }\end{array}$ & $\begin{array}{l}\text { EG:EA + Tropisetron ( } 5 \mathrm{mg} \text {, } \\
\text { intravenous injection prior to the end } \\
\text { of surgery) +routine treatment; CG : } \\
\text { Tropisetron ( } 5 \mathrm{mg} \text {, intravenous } \\
\text { injection prior to the end of } \\
\text { surgery) + routine treatment. }\end{array}$ & $\begin{array}{l}\text { Acupoints: bilateral Neiguan } \\
\text { (PC6) and Zusanli (ST36), mode: } \\
\text { dense-disperse wave; frequency: } \\
2 \mathrm{~Hz} \text {; intensity (mA): maximum } \\
\text { tolerable. }\end{array}$ & $\begin{array}{l}30 \text { minutes prior to } \\
\text { the surgery, once for } \\
30 \mathrm{~min} \text {. }\end{array}$ & PONV, TFF & RCT \\
\hline $\begin{array}{l}\text { Wang et al. } \\
(2018)[30]\end{array}$ & $\begin{array}{l}\text { EG:EA + Tropisetron }(5 \mathrm{mg} \text {, } \\
\text { intravenous injection prior to the end } \\
\text { of surgery) + routine treatment; CG: } \\
\text { Tropisetron ( } 6 \mathrm{mg} \text {, intravenous } \\
\text { injection prior to the end of } \\
\text { surgery) + routine treatment. }\end{array}$ & $\begin{array}{l}\text { Acupoints: bilateral Neiguan } \\
\text { (PC6) and Zusanli (ST36), mode: } \\
\text { dense-disperse wave; frequency: } \\
2 \mathrm{~Hz} \text {; intensity (mA): maximum } \\
\text { tolerable. }\end{array}$ & $\begin{array}{l}24 \text { hours prior to the } \\
\text { surgery, once for } \\
30 \mathrm{~min} .\end{array}$ & TFF, PONV & RCT \\
\hline $\begin{array}{l}\text { Wang et al. } \\
\text { (2011) [31] }\end{array}$ & $\begin{array}{l}\mathrm{EG}: \mathrm{EA}+\text { routine treatment; } \mathrm{CG}: \\
\quad \text { routine treatment. }\end{array}$ & $\begin{array}{l}\text { Acupoints: bilateral Zusanli } \\
\text { (ST36), Neiguan (PC6), mode: } \\
\text { continuous wave; frequency: } \\
\text { 2 Hz; intensity (mA): maximum } \\
\text { tolerable. }\end{array}$ & $\begin{array}{l}\text { Prior to the surgery, } \\
\text { once for } 15 \mathrm{~min} \text {. }\end{array}$ & $\begin{array}{l}\text { PONV, MTL, } \\
\text { VIP, number of } \\
\text { TFF more than } \\
72 \mathrm{~h}\end{array}$ & RCT \\
\hline
\end{tabular}


TABLE 2: Continued.

\begin{tabular}{|c|c|c|c|c|c|}
\hline $\begin{array}{l}\text { First author } \\
\text { (year) }\end{array}$ & Intervention & Intervention parameters & Intervention dose & Main outcome & $\begin{array}{l}\text { Study } \\
\text { design }\end{array}$ \\
\hline $\begin{array}{l}\text { Wang and } \\
\mathrm{Xi}(2017) \\
{[32]}\end{array}$ & $\begin{array}{l}\text { EG : EA + routine treatment; CG: } \\
\text { Ginger partitioned moxibustion on } \\
\text { umbilicus + routine treatment. }\end{array}$ & $\begin{array}{l}\text { Acupoints: bilateral Liangmen } \\
\text { (ST21) and Zusanli (ST36), } \\
\text { Shangjuxu (ST37), Zhongwan } \\
\text { (RN12), Tianshu (ST25), mode: } \\
\text { continuous wave. }\end{array}$ & $\begin{array}{l}\text { After surgery, once } \\
\text { for } 30 \text { min, once a } \\
\quad \text { day, for } 3 \mathrm{~d} \text {. }\end{array}$ & TBS, TFF & RCT \\
\hline $\begin{array}{l}\text { Xi and } \\
\text { Wang } \\
(2015)[33]\end{array}$ & $\begin{array}{l}\text { EG : EA + routine treatment; CG1 : } \\
\text { Ginger partitioned moxibustion on } \\
\text { umbilicus + routine treatment }\end{array}$ & $\begin{array}{l}\text { Acupoints: bilateral Liangmen } \\
\text { (ST21) and Zusanli (ST36), } \\
\text { Shangjuxu (ST37), Zhongwan } \\
\text { (RN12), Tianshu (ST25), mode: } \\
\text { continuous wave; intensity } \\
\text { (mA): maximum tolerable. }\end{array}$ & $\begin{array}{l}\text { After surgery, once } \\
\text { for } 30 \text { min, once a } \\
\text { day, for } 3 \mathrm{~d} \text {. }\end{array}$ & $\begin{array}{l}\text { TBS, TFF, } \\
\text { MTL, GAS, }\end{array}$ & \\
\hline VIP & RCT & & & & \\
\hline $\begin{array}{l}\text { Yang et al. } \\
(2012) \text { [34] }\end{array}$ & $\begin{array}{l}\mathrm{EG}: \mathrm{EA}+\text { routine treatment; CG: } \\
\quad \text { routine treatment. }\end{array}$ & $\begin{array}{l}\text { Acupoints: bilateral Sanyinjiao } \\
\text { (SP6) and Zusanli (ST36), mode: } \\
\text { dense-disperse wave; frequency: } \\
\text { 2/100 Hz; intensity (mA): } \\
\text { maximum tolerable. }\end{array}$ & $\begin{array}{l}30 \text { minutes prior to } \\
\text { the surgery until the } \\
\text { end of surgery. }\end{array}$ & TFF & RCT \\
\hline $\begin{array}{l}\text { Huang et al. } \\
\text { (2021) [35] }\end{array}$ & $\begin{array}{l}\mathrm{EG}: \mathrm{EA}+\text { routine treatment; } \mathrm{CG}: \\
\quad \text { routine treatment. }\end{array}$ & $\begin{array}{l}\text { Acupoints: bilateral Neiguan } \\
\text { (PC6), mode: dense-disperse } \\
\text { wave; frequency: } 3 / 20 \mathrm{~Hz} \text {; } \\
\text { intensity }(\mathrm{mA}) \text { : unclear. }\end{array}$ & $\begin{array}{l}\text { Prior to the surgery, } \\
\text { once for } 20 \mathrm{~min} \text {. }\end{array}$ & $\begin{array}{l}\text { PONV, MTL, } \\
\text { GAS }\end{array}$ & RCT \\
\hline $\begin{array}{l}\text { Ye and } \\
\text { Huang } \\
(2019)[36]\end{array}$ & $\begin{array}{l}\mathrm{EG}: \mathrm{EA}+\text { routine treatment; } \mathrm{CG}: \\
\text { routine treatment. }\end{array}$ & $\begin{array}{l}\text { Acupoints: bilateral Neiguan } \\
\text { (PC6), mode: unreported, } \\
\text { frequency: } 3 / 21 \mathrm{~Hz} \text {; intensity } \\
\text { (mA): comfortable. }\end{array}$ & $\begin{array}{l}\text { Prior to the surgery, } \\
\text { once for } 20 \mathrm{~min} \text {. }\end{array}$ & PONV & RCT \\
\hline $\begin{array}{l}\text { Yu and } \\
\text { Ning }(2016) \\
{[37]}\end{array}$ & $\begin{array}{l}\text { EG: EA + Tropisetron }(2 \mathrm{mg} \text {, } \\
\text { intravenous injection prior to the end } \\
\text { of surgery) + routine treatment; CG1 : } \\
\text { Tropisetron ( } 2 \mathrm{mg} \text {, intravenous } \\
\text { injection prior to the end of } \\
\text { surgery) + routine treatment. }\end{array}$ & $\begin{array}{l}\text { Acupoints: bilateral Neiguan } \\
\text { (PC6), and Hegu (LI4), mode: } \\
\text { dense-disperse wave; frequency: } \\
\text { 20/100 Hz; intensity (mA): } \\
10 \mathrm{~mA} \text {. }\end{array}$ & $\begin{array}{l}\text { Prior to the surgery, } \\
\text { once for } 30 \mathrm{~min} \text {. }\end{array}$ & PONV & RCT \\
\hline $\begin{array}{l}\text { Zhang et al. } \\
(2013) \text { [38] }\end{array}$ & $\begin{array}{l}\mathrm{EG}: \mathrm{EA}+\text { routine treatment; } \mathrm{CG}: \\
\quad \text { routine treatment. }\end{array}$ & $\begin{array}{l}\text { Acupoints: bilateral Liangqiu } \\
\text { (ST34) and Zusanli (ST36), } \\
\text { mode: continuous wave; } \\
\text { frequency: } 2 \mathrm{~Hz} \text {; intensity (mA): } \\
\text { maximum tolerable. }\end{array}$ & $\begin{array}{l}30 \text { minutes prior to } \\
\text { the surgery until the } \\
\text { end of surgery. }\end{array}$ & $\mathrm{pHi}, \mathrm{PgCO}_{2}$ & RCT \\
\hline
\end{tabular}

EG: electroacupuncture group; CG: control group; EA: electroacupuncture; TFF: time to first flatus; TFD: time first to defecation; TFBS: time to first bowel sound; PONV: postoperative nausea and vomiting; MTL: motilin; GAS: gastrin; $\mathrm{pHi}$ : $\mathrm{PH}$ value of gastric mucosa; $\mathrm{PgCO}_{2}$ : gastric mucosal partial pressure of carbon dioxide; VIP: vasoactive intestinal peptide; and RCT: randomized controlled trials.

30 min prior to surgery $(\mathrm{OR}=0.46,95 \% \mathrm{CI}:[0.28,0.76]$, $\left.P=0.03, I^{2}=0 \%\right)$, or after surgery $(\mathrm{OR}=0.03,95 \% \mathrm{CI}:[0.13$, 0.67], $\left.P=0.003, I^{2}=0 \%\right)$. However, there was no significant difference in the ratio of PONV at postoperative $48 \mathrm{~h}$ between the EG and $\mathrm{CG}$ regardless of the preoperative $\left(\mathrm{OR}=0.64,95 \% \mathrm{CI}:[0.10,4.15], P=0.64, I^{2}=\right.$ not applicable) or postoperative electroacupuncture treatment $\left(\mathrm{OR}=0.52,95 \% \mathrm{CI}:[0.16,1.71], P=0.28, I^{2}=0 \%\right)$.

(2) Motilin (MTL). Two studies [23, 35] investigated the effects of EA on MTL at postoperative $6 \mathrm{~h}$, and four studies $[23,31,33,35]$ evaluated the effect at postoperative $24 \mathrm{~h}$. Meta-analysis results suggested that a significant effect in favor of EA on level of MTL at postoperative $6 \mathrm{~h}(\mathrm{SMD}=-0.93,95 \% \mathrm{CI}:[-1.36,-0.61]$, $P<0.0001, I^{2}=21 \%$ ), while no significant effect was observed at postoperative $24 \mathrm{~h}(\mathrm{SMD}=-0.43,95 \% \mathrm{CI}$ : $\left.[-0.89,0.02], P=0.06, I^{2}=69 \%\right)$ in the EG when compared to the CG (Figure 8 ).

(3) Gastrin (GAS). Two studies [23, 35] compared patients receiving EA to those in the control condition in terms of GAS at postoperative $6 \mathrm{~h}$, and three studies [23, 33, 35] estimated the effect of EA on GAS at postoperative $24 \mathrm{~h}$. Meta-analysis results revealed that no significant effect on GAS was observed at neither postoperative $6 \mathrm{~h}(\mathrm{SMD}=0.20$, 95\% CI: $\left.[-1.62,2.01], \quad P=0.83, I^{2}=96 \%\right)$ nor $24 \mathrm{~h}$ $\left(\mathrm{SMD}=0.63,95 \% \mathrm{CI}:[-0.57,1.84], P=0.30, I^{2}=94 \%\right)$ (Figure 9).

(4) Vasoactive Intestinal Peptide (VIP). Meta-analysis of two studies [31, 33] demonstrated a small, but nonsignificant, overall effect concerning VIP $(\mathrm{SMD}=0.12$, 95\% CI: $[-0.26,0.50], P=0.53, I^{2}=20 \%$ ) (Figure 10). 


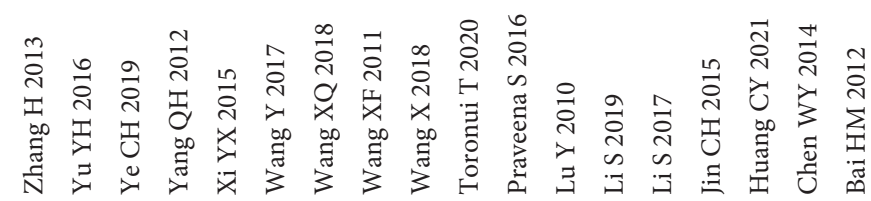

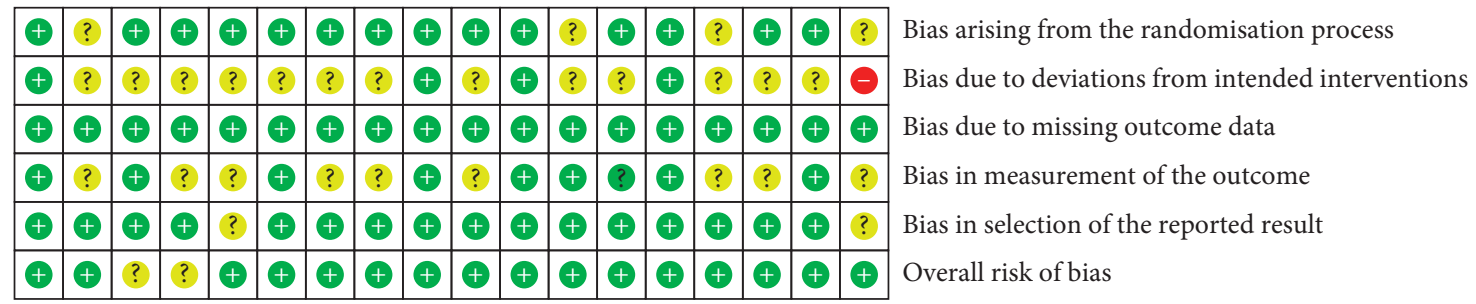

Bias arising from the randomisation process Bias due to deviations from intended interventions

Bias due to missing outcome data Bias in measurement of the outcome

Bias in selection of the reported result

Overall risk of bias
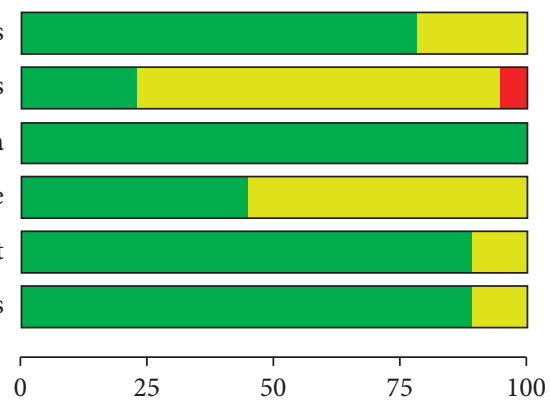

(\%)

$\square$ Low risk of bias

$\square$ Unclear risk of bias

$\square$ High risk of bias

FIgURE 2: Risk of bias graph.

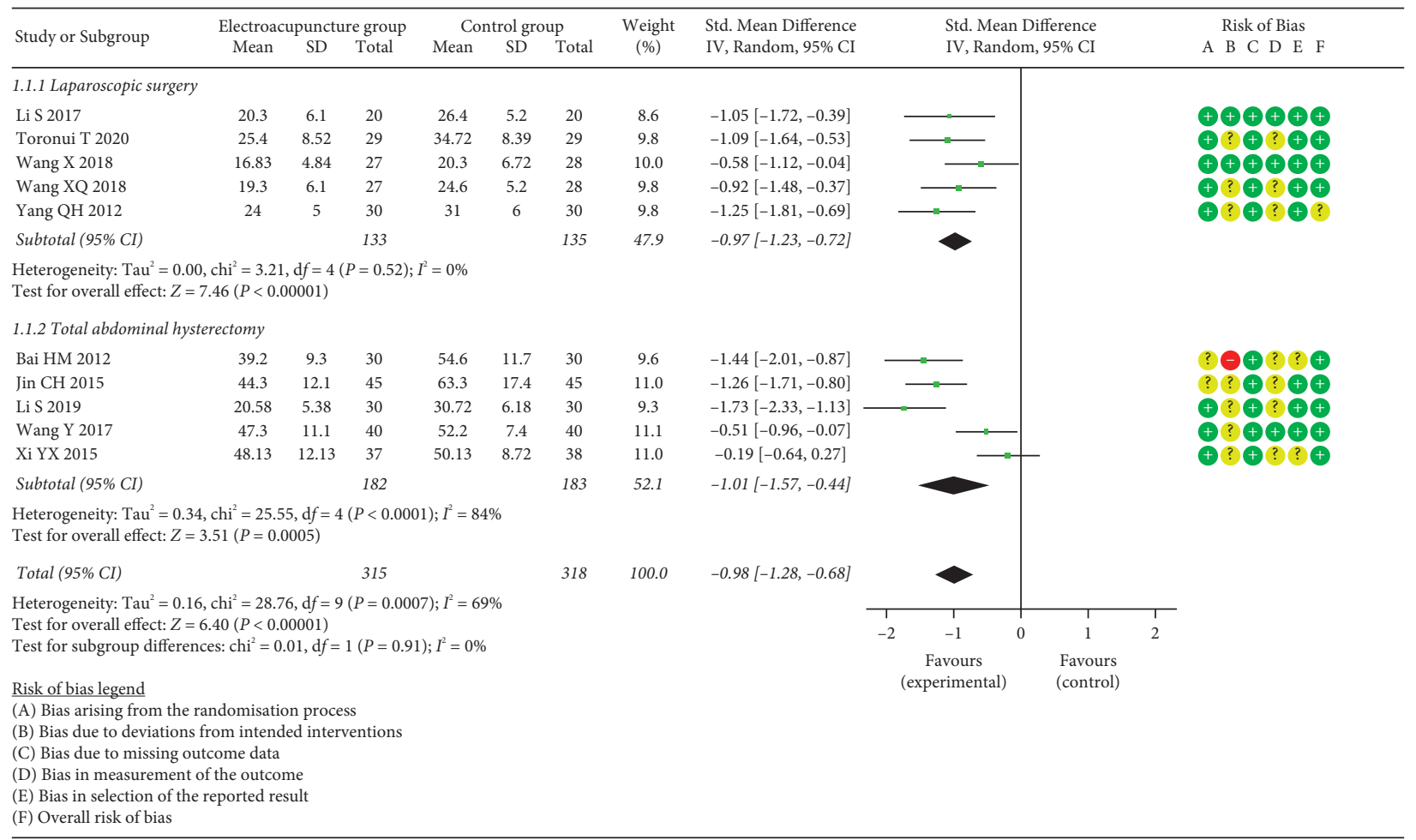

Figure 3: Meta-analysis and forest plot for time to first flatus. 


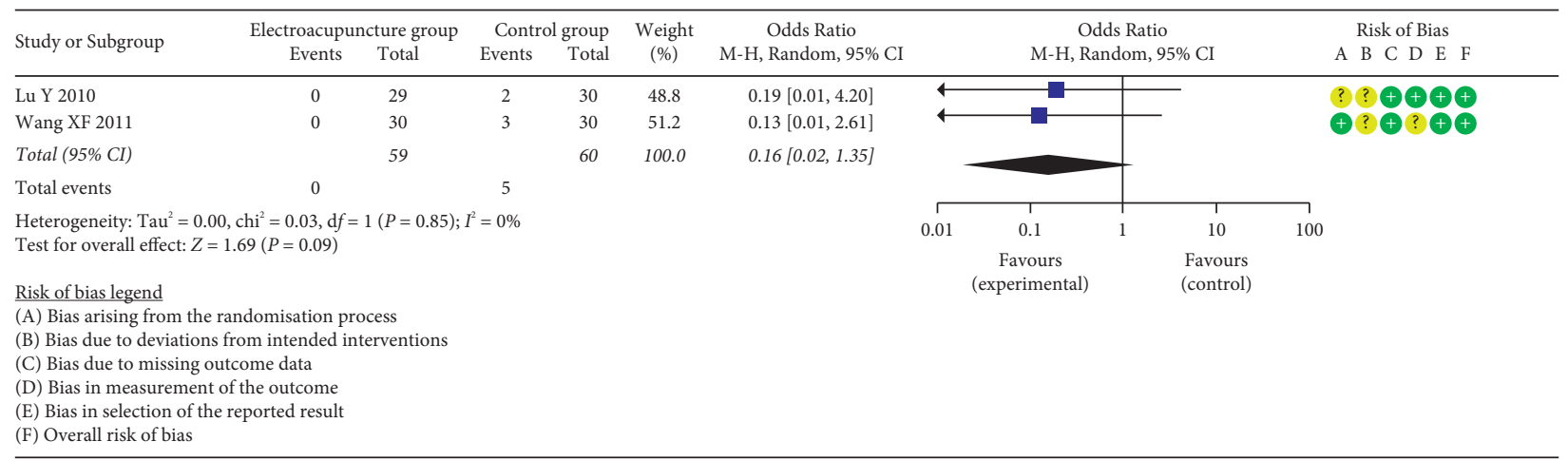

FIGURE 4: Meta-analysis and forest plot for ratio of time to first flatus $>72 \mathrm{~h}$.

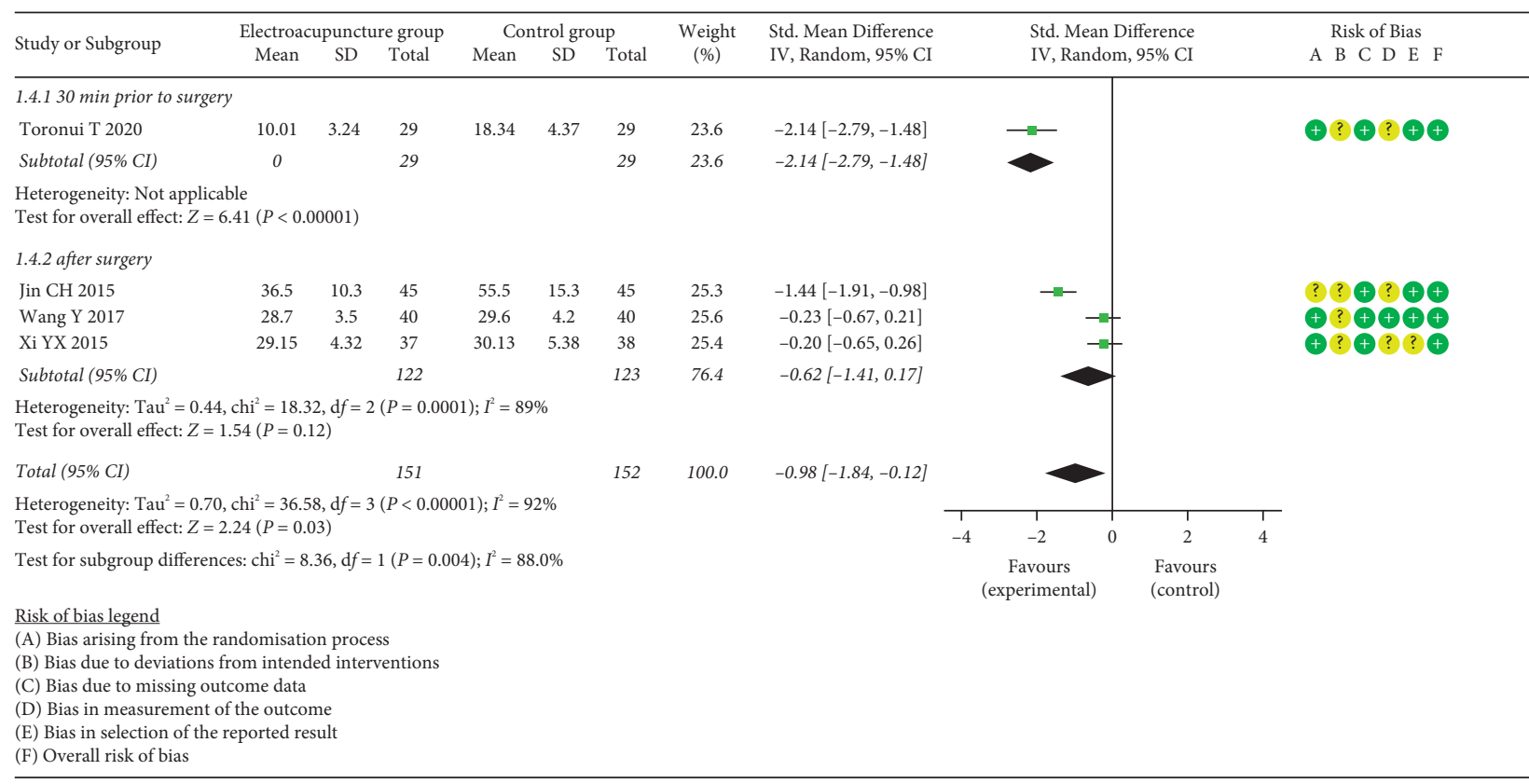

Figure 5: Meta-analysis and forest plot for time to bowel sounds recovery.

\begin{tabular}{|c|c|c|c|c|c|c|c|c|c|c|c|}
\hline \multirow{3}{*}{$\begin{array}{l}\text { Study or Subgroup } \\
\text { Jin CH } 2015\end{array}$} & \multicolumn{3}{|c|}{ Electroacupuncture group } & \multicolumn{3}{|c|}{ Control group } & \multirow{3}{*}{$\begin{array}{c}\begin{array}{c}\text { Weight } \\
(\%)\end{array} \\
60.3\end{array}$} & \multirow{3}{*}{$\begin{array}{l}\text { Std. Mean Difference } \\
\text { IV, Random, 95\% CI } \\
-1.27[-1.73,-0.82]\end{array}$} & \multirow{2}{*}{\multicolumn{2}{|c|}{$\begin{array}{l}\text { Std. Mean Difference } \\
\text { IV, Random, 95\% CI }\end{array}$}} & \multirow{3}{*}{\begin{tabular}{c}
\multicolumn{2}{c}{ Risk of Bias } \\
A B C D E F \\
$? ? \odot ?+\odot$
\end{tabular}} \\
\hline & \multirow{2}{*}{$\begin{array}{c}\text { Mean } \\
50.4\end{array}$} & \multirow{2}{*}{$\frac{\mathrm{SD}}{11.8}$} & \multirow{2}{*}{$\begin{array}{c}\text { Total } \\
45\end{array}$} & \multirow{2}{*}{$\frac{\text { Mean }}{67.3}$} & \multirow{2}{*}{$\frac{\mathrm{SD}}{14.4}$} & \multirow{2}{*}{$\begin{array}{c}\text { Total } \\
45\end{array}$} & & & & & \\
\hline & & & & & & & & & - & & \\
\hline Toronui T 2020 & 35.98 & 6.88 & 29 & 44.38 & 7.21 & 29 & 39.7 & $-1.18[-1.74,-0.62]$ & - & & $\oplus ?+? \oplus \odot$ \\
\hline Total $(95 \%$ CI $)$ & & & 74 & & & 74 & 100.0 & $-1.23[-1.59,-0.88]$ & $\diamond$ & & \\
\hline $\begin{array}{l}\text { Heterogeneity: } \mathrm{Tau}^{2} \\
\text { Test for overall effec }\end{array}$ & $\begin{array}{l}\mathrm{chi}^{2}=0.07 \\
.85(P<0 .\end{array}$ & $\begin{array}{l}\mathrm{d} f=1 \\
0001)\end{array}$ & $P=0.79)$; & $2=0 \%$ & & & & & $\begin{array}{cc} & 1 \\
-4 & -2\end{array}$ & $\begin{array}{ll}1 & 1 \\
2 & 4\end{array}$ & \\
\hline $\begin{array}{l}\text { Risk of bias legend } \\
\text { (A) Bias arising from } \\
\text { (B) Bias due to devia } \\
\text { (C) Bias due to miss } \\
\text { (D) Bias in measure } \\
\text { (E) Bias in selection } \\
\text { (F) Overall risk of bi }\end{array}$ & $\begin{array}{l}\text { ndomisati } \\
\text { rom inten } \\
\text { come data } \\
\text { the outco } \\
\text { eported re }\end{array}$ & $\begin{array}{l}\text { n proce } \\
\text { ed inter } \\
\text { ue } \\
\text { ult }\end{array}$ & $\begin{array}{l}\text { ss } \\
\text { ventions }\end{array}$ & & & & & & $\begin{array}{c}\text { Favours } \\
\text { (experimental) }\end{array}$ & $\begin{array}{l}\text { Favours } \\
\text { (control) }\end{array}$ & \\
\hline
\end{tabular}

Figure 6: Meta-analysis and forest plot for time to first defecation.

(5) $\mathrm{pH}$ Value of Gastric Mucosa ( $\mathrm{pHi}$ ). Meta-analysis of two studies $[24,38]$ indicated that no significant difference in pHi was determined between the two groups neither at pneumoperitoneum for $30 \mathrm{~min}(\mathrm{SMD}=0.70,95 \% \mathrm{CI}$ : $\left.[-0.47,1.88], P=0.24, I^{2}=88 \%\right)$ nor at $30 \mathrm{~min}$ after the end of pneumoperitoneum $(\mathrm{SMD}=1.15,95 \% \mathrm{CI}$ : $[-0.95,3.24]$, $\left.P=0.28, I^{2}=96 \%\right)$ (Figure 11$)$.

(6) Gastric Mucosal Partial Pressure of Carbon Dioxide $\left(\mathrm{PgCO}_{2}\right)$. Two studies examined the effect of EA on $\mathrm{PgCO}_{2}$. 


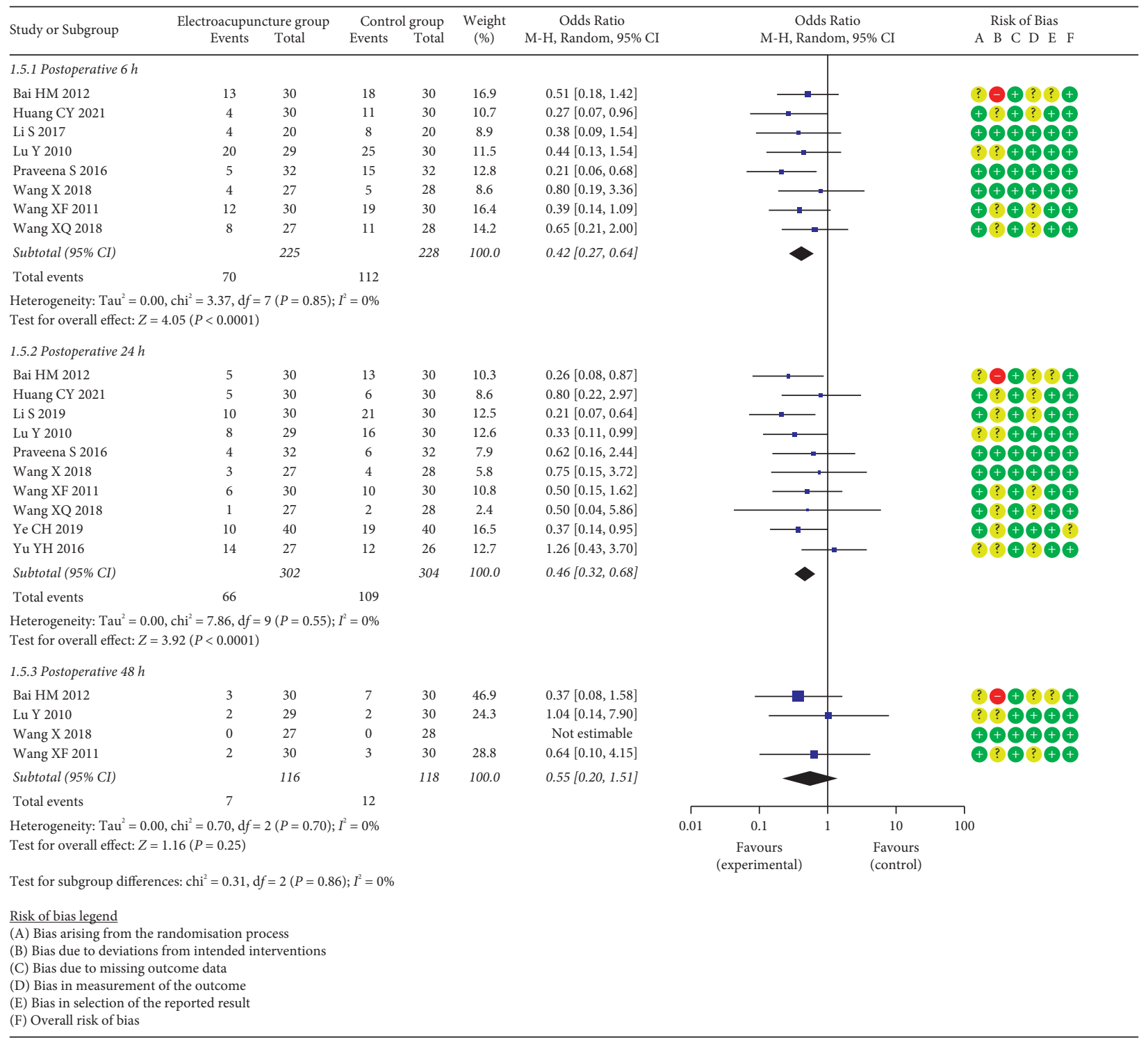

Figure 7: Meta-analysis and forest plot for ratio of PONV.

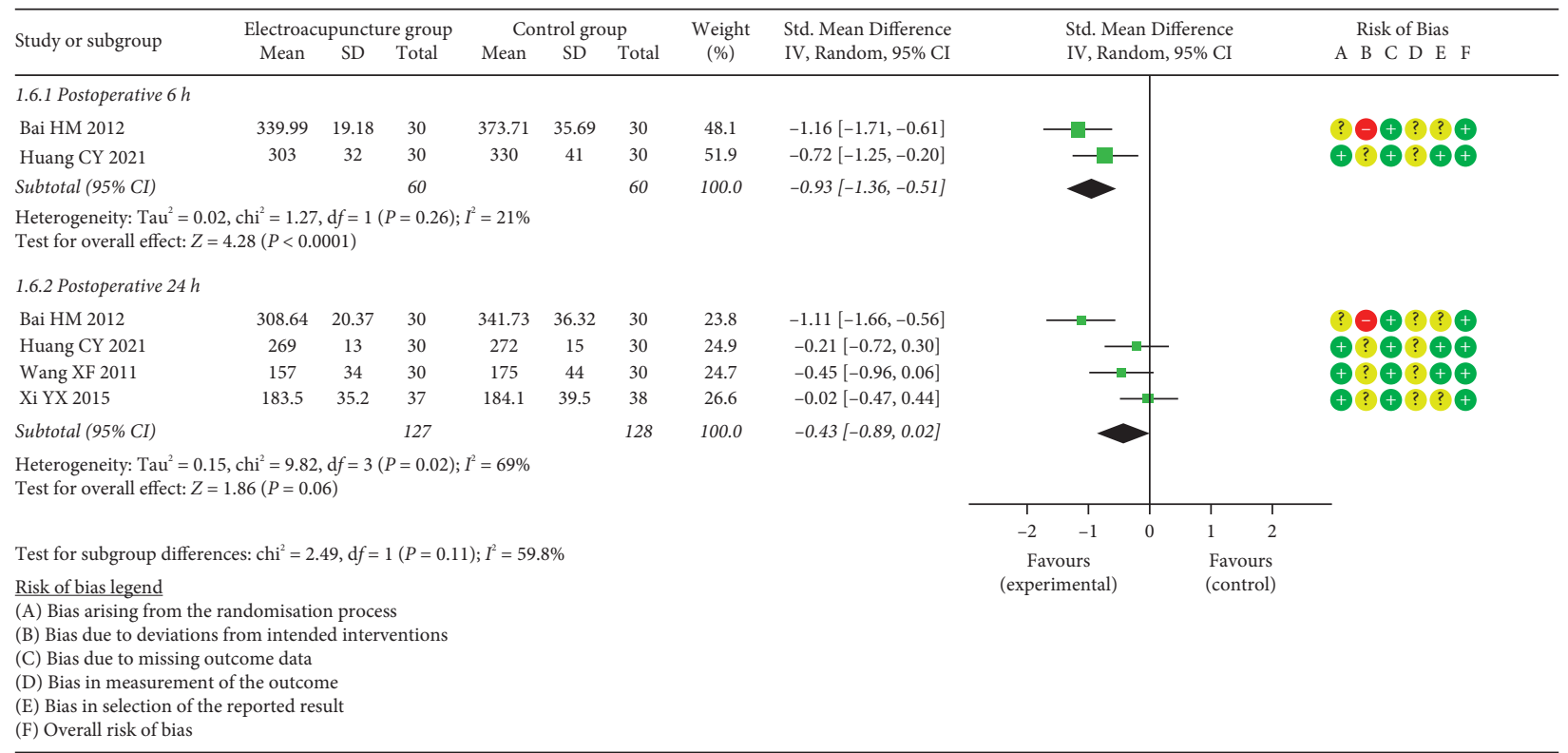




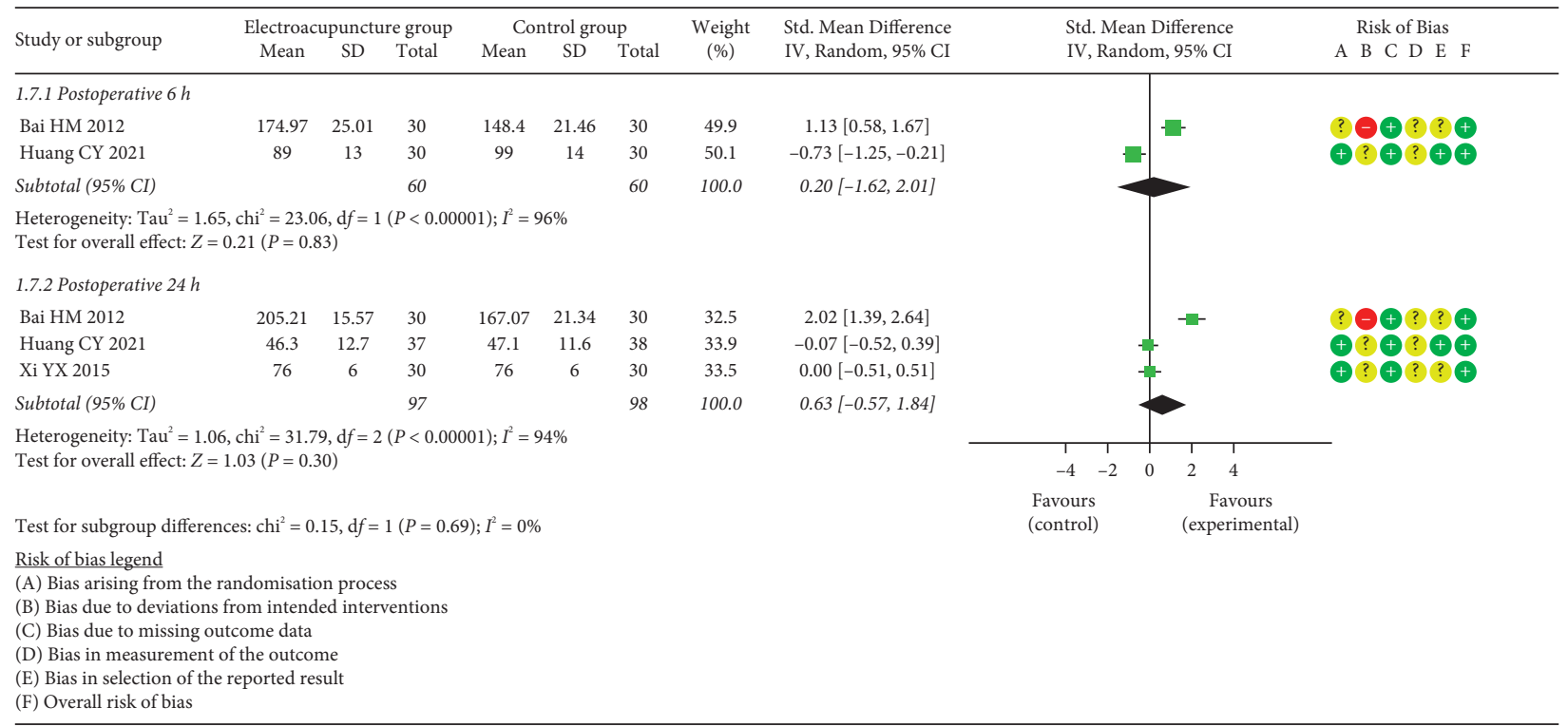

Figure 9: Meta-analysis and forest plot for gastrin.

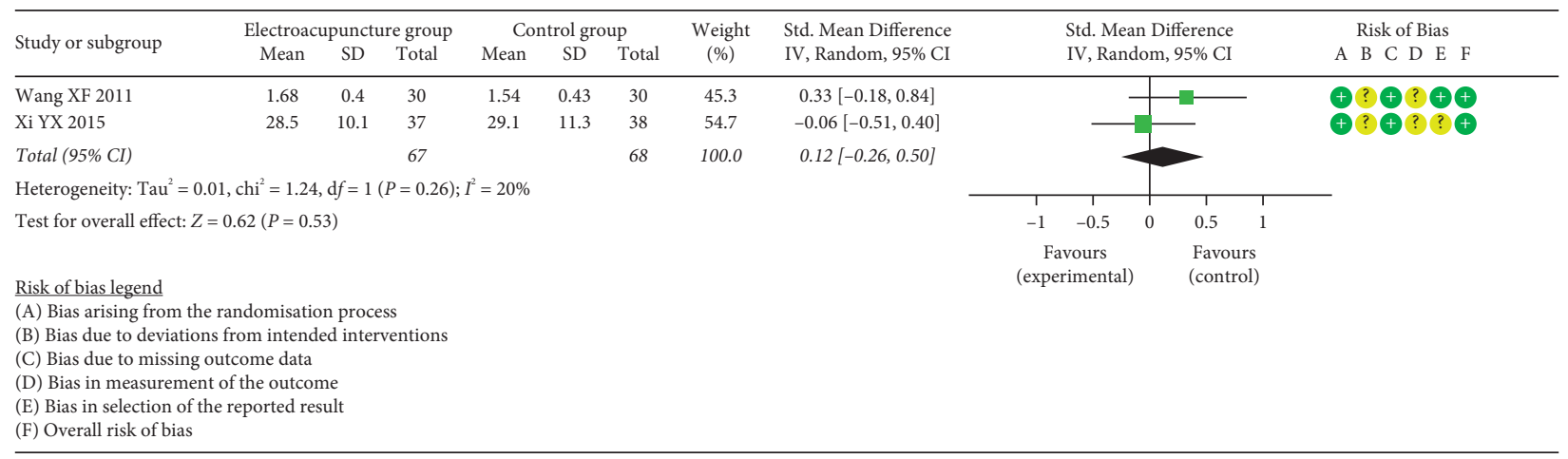

Figure 10: Meta-analysis and forest plot for VIP.

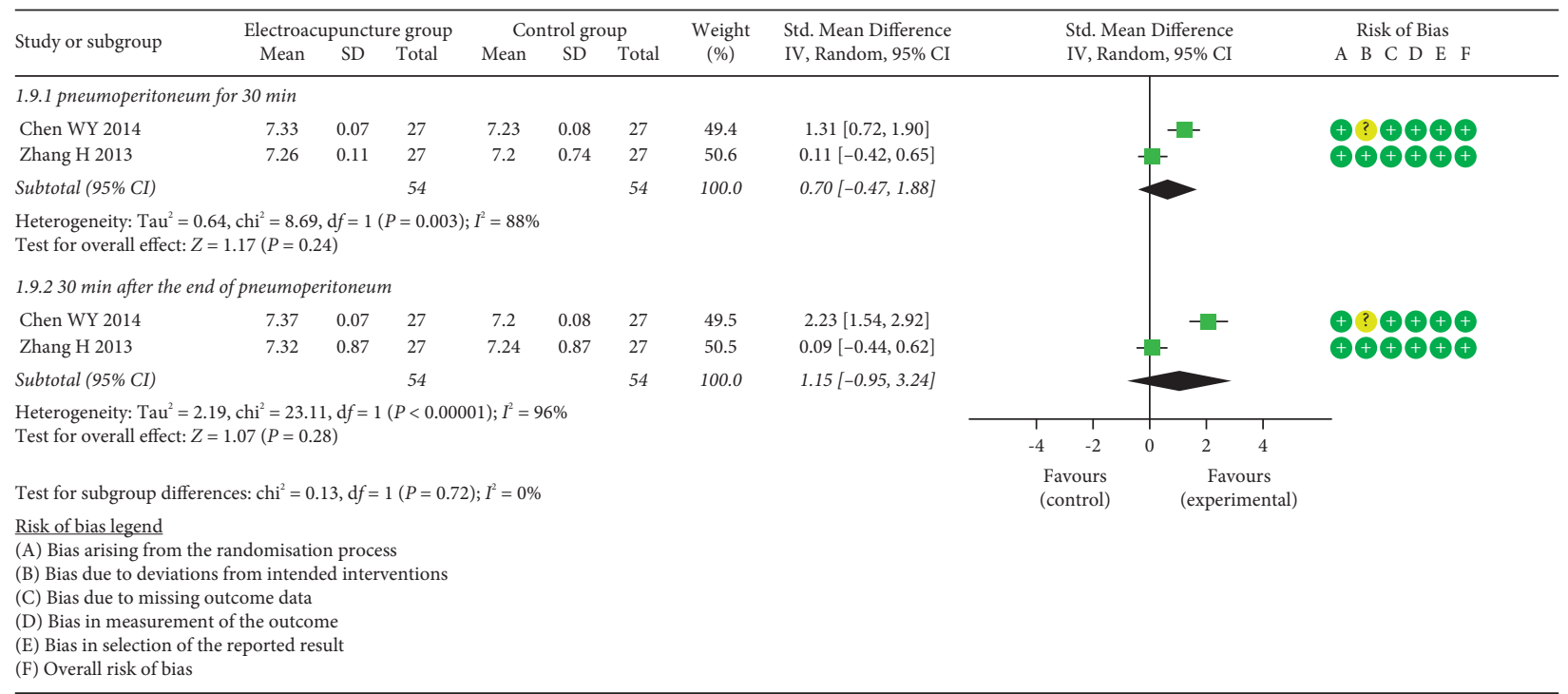

FIgURE 11: Meta-analysis and forest plot for $\mathrm{pHi}$. 


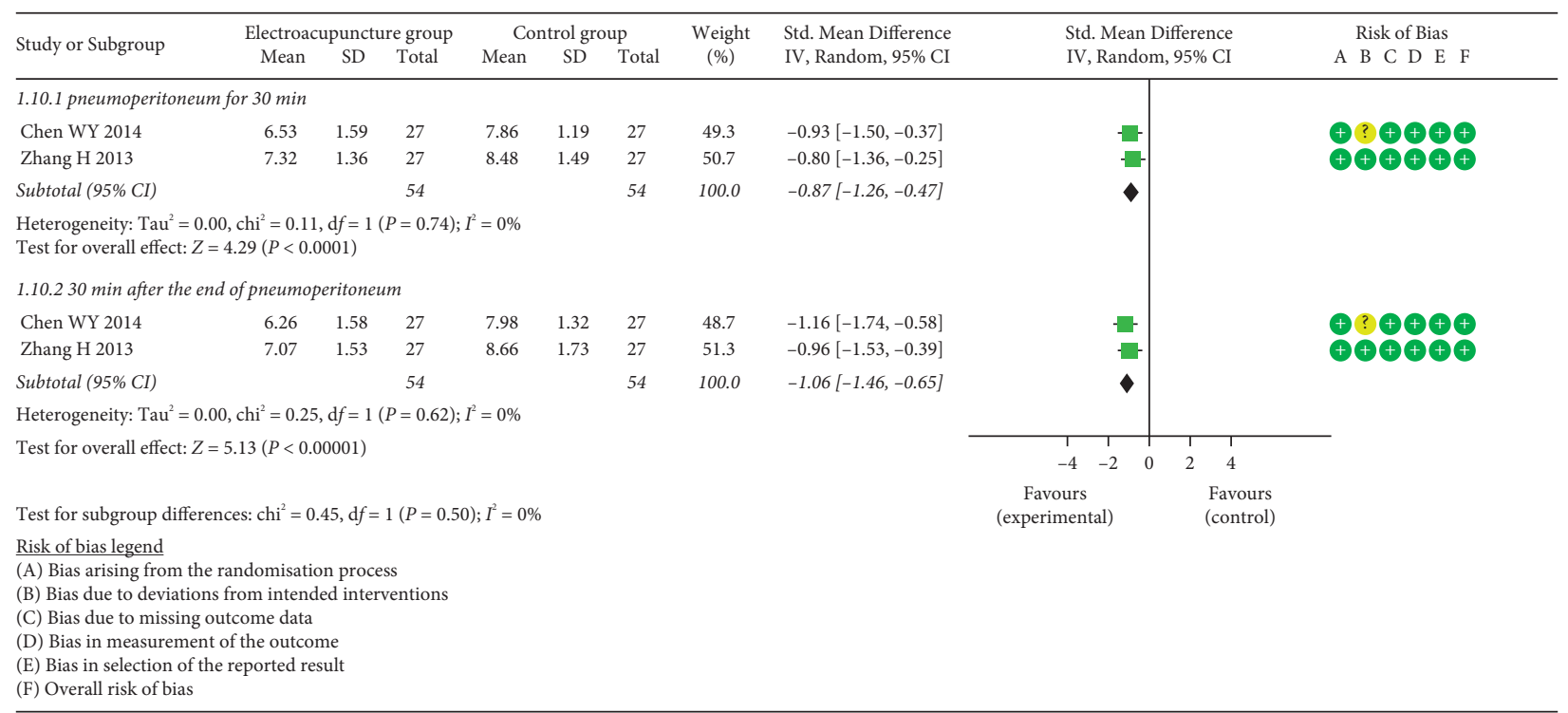

FIgURE 12: Meta-analysis and forest plot for $\mathrm{PgCO}_{2}$.

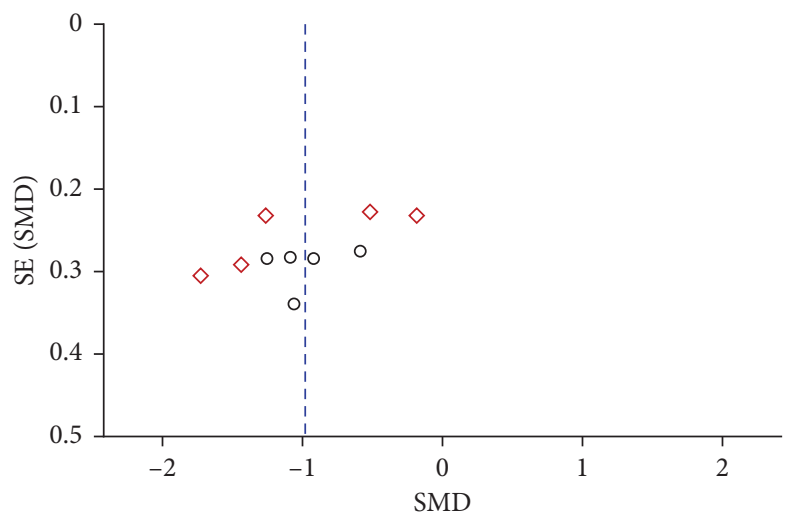

Subgroups

○ Laparoscopic surgery

$\diamond$ Total abdominal hysterectomy

(a)

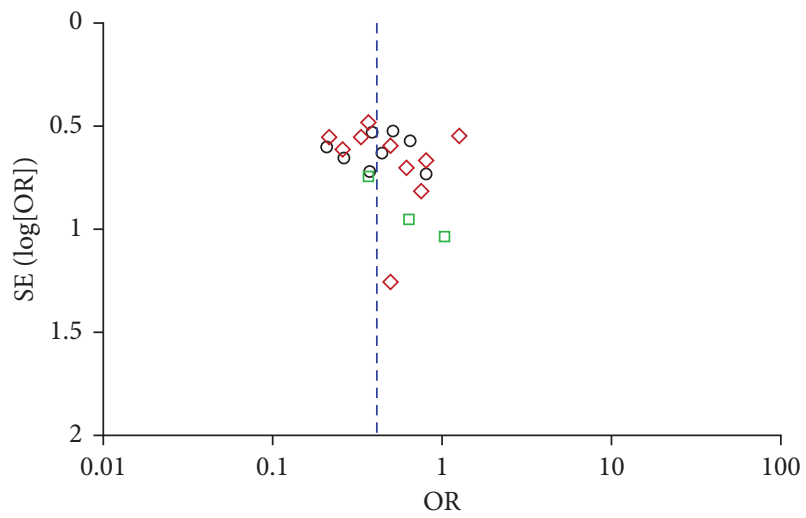

Subgroups
$\circ$ Postoperative $6 \mathrm{~h}$
$\diamond$ Postoperative $24 \mathrm{~h}$
$\square$ Postoperative $48 \mathrm{~h}$

FIgure 13: Publication bias assessed by funnel plots for TFF (a) and PONV (b).

Meta-analysis results revealed that a large significant effect on decreasing $\mathrm{PgCO}_{2}$ was found in the EG VS CG, both at pneumoperitoneum for $30 \mathrm{~min}(\mathrm{SMD}=-0.87,95 \% \mathrm{CI}$ : $\left.[-1.26,-0.47], P<0.0001, I^{2}=0 \%\right)$ and $30 \mathrm{~min}$ after the end of pneumoperitoneum $(\mathrm{SMD}=-1.06,95 \% \mathrm{CI}$ : $[-1.46$, -0.65 ], $P<0.00001, I^{2}=0 \%$ ) (Figure 12).

(7) Hospital Stay. In the study, hospital stay was recorded in only one study [30], which reported that electroacupuncture applied at $24 \mathrm{~h}$ prior to surgery showed no significant effect on the duration of hospital stay (EG vs. CG: $(3.6 \pm 0.4) d$ vs. $(3.9 \pm 0.2) d, P=0.492)$.

(8) Adverse Event. One participant in one study [21] reported pain at the needling sites and bruising, while the side effects were alleviated spontaneously without any medical assistance. In addition, it was reported in two studies $[30,38]$ that three participants withdrew because they were not intolerant to EA.

3.5. Sensitivity Analysis. In this study, we conducted the sensitivity analysis to evaluate the robustness of primary outcome indicators with high heterogeneity by removing studies from the analysis individually. After sensitivity analysis for TFF, no substantial change about overall heterogeneities and results was detected (Supplementary Table 2). Meanwhile, we performed sensitivity analysis for TBS, and the results were listed in Supplementary Table 3. The overall effect indicated no statistically significant difference 
when the study reported by Jin and Jing [25] (SMD $=-0.83$, 95\% CI: [-1.09, 0.24], $\left.P=0.13, I^{2}=93 \%\right)$ or Toronui [28] $\left(\mathrm{SMD}=-0.62,95 \% \mathrm{CI}\right.$ : $\left.[-1.41,0.17], P=0.12, I^{2}=89 \%\right)$ was eliminated. However, the results of subgroup analysis by intervention time points for the remaining studies $[28,32,33]$ showed no substantial change. It was found that TBS was significantly shorter in the EG than that in the CG when EA was received prior to surgery than that in the CG $\left(\mathrm{SMD}=-2.14,95 \% \mathrm{CI}:[-2.79,-1.48], P<0.00001, I^{2}=\right.$ not applicable), whereas no significant difference was detected with no heterogeneity when EA was applied after surgery $\left(\mathrm{SMD}=-0.22,95 \% \mathrm{CI}:[-0.53,0.10], P=0.18, I^{2}=0 \%\right)$.

3.6. Publication Bias. In the present study, Begg's test and Egger's test were employed to examine publication bias for the indices recorded more than 2 included trails. It was suggested that there should be no publication bias except for MTL at postoperative $24 \mathrm{~h}(P=0.042)$ (Supplementary Table 4). Moreover, visual inspection of funnel plots seemed to be relatively symmetrical for TFF and PONV (Figure 13), indicating no evidence for publication bias.

3.7. Evidence Quality Assessment According to GRADE. Based on GRADE guidelines, we evaluated the quality of evidence from five aspects, including risk of bias, inconsistency, indirectness, imprecision, and publication bias. During the treatment period, EA therapists directly contacted to participants, which led to the high risk of performance bias, and consequently lowered the evidence quality. In this study, there was low evidence in TFF, TFD and PONV, and very low evidence in the remaining. Details were recorded in Supplementary Table 5.

\section{Discussion}

In spite of continuous improvement for surgical approach and technique, postoperative gastrointestinal dysfunction still commonly occurred after gynecological surgery. To resolve this clinical concern, EA has been proposed to promote gastrointestinal function recovery, while relevant evidence is sparse. To the best of our knowledge, this is first systematical review and meta-analysis regarding the efficacy and safety of EA on recovery of gastrointestinal function for patients receiving gynecological surgery.

We found that EA was able to shorten the time to first flatus, first defecation after gynecological surgery. These findings were in accordance with those reported by the previous meta-analyses [11-14] for other patient populations, but there were some new findings after subgroup analysis in the current study. Our findings suggested that EA was beneficial to reduce the time to first of bowel sounds recovery when EA was used at $30 \mathrm{~min}$ prior to surgery. However, no significant effect on TBS was observed when patients undergoing gynecological surgery received EA treatment after surgery. Additionally, the evidence in favor of EA for decreasing the ratio of PONV was observed only at postoperative $6 \mathrm{~h}$ and $24 \mathrm{~h}$, which echo the results reported by Lee et al. [39], while the benefits were unobvious at postoperative $48 \mathrm{~h}$. Similarly, the effect of EA on MTL was detected only within postoperative $6 \mathrm{~h}$. Thus, our findings showed that the benefits of EA for PONV and MTL concentrate within $24 \mathrm{~h}$ after surgery, and even earlier. MTL stimulated gastrointestinal motility and accelerated gastric emptying, which could induce or aggravate nausea and vomiting [40, 41]. Thus, it could be the reason that EA decreased MTL level and consequently resulted in lowering the ratio of PONV at with postoperative $24 \mathrm{~h}$. After subgroup analysis for PONV by intervention points, we found that EA presented a largest effect in lowering the ratio of PONV when it was applied at $30 \mathrm{~min}$ prior to surgery, whereas no or very small effect was found when patients were treated with EA after gynecological surgery. It might be the reason that factors contributing to postoperative gastrointestinal dysfunction mainly generated during intraoperative period; it should be more proper to applied EA before the start of surgery until the end of surgery, while the effectiveness of EA would be weakened when used too early, and postoperative application seems to be late.

$\mathrm{PgCO}_{2}$ is a sensitive indicator reflecting the changes of blood oxygen in gastric mucosa [42]. It was proved that $\mathrm{pHi}$ was closely related with intestinal oxygen consumption, and decreased gastric pHi level prognosticated morbidity and mortality [43]. The results of this meta-analysis revealed that EA deceased the $\mathrm{PgCO}_{2}$ both at $30 \mathrm{~min}$ after the start of pneumoperitoneum and $30 \mathrm{~min}$ after the end of pneumoperitoneum, whereas it was ineffective for mediation of $\mathrm{pHi}$ after surgery. Additionally, GAS was the hormone primarily responsible for gastric acid secretion [44], and VIP was a gut peptide hormone regulating gut motility [45]; hence, they were associated with postoperative gastrointestinal function [46]. However, EA showed no significant effect on GAS and VIP after gynecological surgery. Therefore, EA accelerated the recovery of postoperative gastrointestinal function through other pathways, not depending on the mediation of GAS or VIP.

Several potential limitations should be considered in the present review had. Firstly, the EA parameters including acupoints, and times and frequency of electroacupuncture were selected without a consolidated standard in the included studies, which could be a potential source of clinical heterogeneity; secondly, blind methods of the included studies were rarely provided in detail; thirdly, as was reported in the previous basic experimental studies, EA mediated of the autonomic nervous system, improved dysmotility and local inflammation, and consequently ameliorated POI to restore gastrointestinal function [47], and the therapeutic effects was different when using lower limb and abdomen acupoints [48]. However, few clinical trials focus on examining the different effect of EA by using different acupoints.

Given the limitations of this work, large-scale RCTs with more rigorous and robust methods are still needed in future studies. Most importantly, the majority of the studies were not blind to participants and acupuncturists; sham-controlled studies should be performed to avoid performance bias. Hence, more studies should be conducted strictly following standard reporting guidelines such as CONSORT 
[49]. Furthermore, more RCTs in the future should investigate the therapeutic effects of EA using different acupoints in order to identify the definitely effective acupoints.

\section{Conclusion}

In this analysis, we systematically reviewed and quantified the effect of EA on gastrointestinal function after gynecological surgery. Overall, EA was an effective and safe treatment for promoting recovery of postoperative gastrointestinal function, such as shortening TFF and TFD, TBS, regulating MTL, and decreasing the ratio of PONV within postoperative 24h, for patients receiving gynecological surgery through abdominal and laparoscopic approaches, while the effects on MTL and PONV varied with different intervention points, and EA used at $30 \mathrm{~min}$ prior to surgery might be recommended. Moreover, EA could regulate $\mathrm{PgCO}_{2}$ during anesthesia process, which was associated with the recovery of postoperative gastrointestinal function. However, EA exerted no significant impact on mediating GAS, VIP, and $\mathrm{pHi}$. Thus, EA could be a promising strategy for the prevention and treatment of gastrointestinal dysfunction after gynecological surgery. Notably, evidence quality ranged from low to very low; large-scale and high-quality RCTs were needed.

\section{Data Availability}

The original data analyzed in this study are presented in the article; further inquiries should be directed to the corresponding authors.

\section{Conflicts of Interest}

The authors declare that they have no conflicts of interest regarding the publication of this paper.

\section{Authors' Contributions}

Y-G and X-G designed the study. X-G and YuZ-Z performed the literature searches and designed the data-extraction form. X-G and YuZ-Z selected the studies. X-G and YuZ-Z extracted the data. X-G and YT-K completed the statistical analyses. Y-G, YiZ-Z, and YT-K revised the manuscript.

\section{Supplementary Materials}

Supplementary file 1: Supplementary Figure 1. The search strategy in this review. Supplementary Figure 2. Forest plot of time to first flatus by subgroup analysis for anesthesia type. Supplementary Figure 3. Forest plot of time to first flatus by subgroup analysis for intervention points. Supplementary Figure 4. Forest plot of time to first flatus by subgroup analysis for comparators. Supplementary Figure 5. Forest plot of time to bowel sounds recovery by subgroup analysis for comparators. Supplementary Table 1. Sensitivity analysis for time to first flatus. Supplementary Table 1. PEDro scores of the included studies. Supplementary Table 2. Sensitivity analysis for time to first flatus. Supplementary Table 3. Evidence quality assessment according to GRADE. Supplementary file 2. PRISMA checklist. (Supplementary Materials)

\section{References}

[1] Q. Zhou, L. Cao, D. Wen, and Z. Chen, "The assessment on influencing factors for postoperative recovery of gastrointestinal function based on the gynecological abdominal surgery," in Proceedings of the 2012 IEEE International Conference on Bioinformatics and Biomedicine Workshops, pp. 472-476, Philadelphia, PA, USA, October 2012.

[2] Z. L. Li, B. C. Zhao, W. T. Deng et al., "Incidence and risk factors of postoperative ileus after hysterectomy for benign indications," International Journal of Colorectal Disease, vol. 35, no. 11, pp. 2105-2112, 2020.

[3] F. Oyama, M. Futagami, T. Shigeto et al., "Preventive effect of daikenchuto, a traditional Japanese herbal medicine, on onset of ileus after gynecological surgery for malignant tumors," Asia-Pacific Journal of Clinical Oncology, vol. 16, no. 4, pp. 254-258, 2020.

[4] J. Fanning and R. Hojat, "Safety and efficacy of immediate postoperative feeding and bowel stimulation to prevent ileus after major gynecologic surgical procedures," Journal of the American Osteopathic Association, vol. 111, no. 8, pp. 469-472, 2011.

[5] A. Nanthiphatthanachai and P. Insin, "Effect of chewing gum on gastrointestinal function recovery after surgery of gynecological cancer patients at rajavithi hospital: a randomized controlled trial," Asian Pacific Journal of Cancer Prevention, vol. 21, no. 3, pp. 761-770, 2020.

[6] D. Ruan, J. Li, J. Liu et al., "Acupoint massage can effectively promote the recovery of gastrointestinal function after gynecologic laparoscopy," Journal of Investigative Surgery, vol. 34, no. 1, pp. 91-95, 2021.

[7] D. Ruan, J. Li, J. Liu et al., "Acupoint massage can effectively promote the recovery of gastrointestinal function after gynecologic laparoscopy," Journal of Investigative Surgery, vol. 34, no. 1, pp. 91-95, 2021.

[8] H. Li, T. He, Q. Xu et al., "Acupuncture and regulation of gastrointestinal function," World Journal of Gastroenterology, vol. 21, no. 27, pp. 8304-8313, 2015.

[9] S. S. Ng, W. W. Leung, S. S. Hon, J. C. Li, C. Y. Wong, and J. F. Lee, "Electroacupuncture for ileus after laparoscopic colorectal surgery: a randomised sham-controlled study," Hong Kong Medical Journal, vol. 19, no. S9, pp. 33-35, 2013.

[10] Z. Q. Meng, M. K. Garcia, J. S. Chiang et al., "Electro-acupuncture to prevent prolonged postoperative ileus: a randomized clinical trial," World Journal of Gastroenterology, vol. 16, no. 1, pp. 104-111, 2010.

[11] K. B. Cheong, J. Zhang, and Y. Huang, "Effectiveness of acupuncture in postoperative ileus: a systematic review and Meta-analysis," Journal of Traditional Chinese Medicine, vol. 36, no. 3, pp. 271-282, 2016.

[12] Y. H. Liu, G. T. Dong, Y. Ye et al., "Effectiveness of acupuncture for early recovery of bowel function in cancer: a systematic review and meta-analysis," Evidence-Based Complementary and Alternative Medicine, vol. 2017, Article ID 2504021, 15 pages, 2017.

[13] Y. Liu, B. H. May, A. L. Zhang et al., “Acupuncture and related therapies for treatment of postoperative ileus in colorectal cancer: a systematic review and meta-analysis of randomized controlled trials," Evidence-Based Complementary and Alternative Medicine, vol. 2018, Article ID 3178472, 18 pages, 2018.

[14] K. B. Chen, Y. Huang, X. L. Jin et al., "Electroacupuncture or transcutaneous electroacupuncture for postoperative ileus after abdominal surgery: a systematic review and meta- 
analysis," International Journal of Surgery, vol. 70, pp. 93-101, 2019.

[15] M. J. Page, J. E. McKenzie, P. M. Bossuyt et al., "The PRISMA 2020 statement: an updated guideline for reporting systematic reviews," BMJ, vol. 372, p. n71, 2021.

[16] J. A. C. Sterne, J. Savović, M. J. Page et al., "RoB 2: a revised tool for assessing risk of bias in randomised trials," $B M J$, vol. 366, p. 14898, 2019.

[17] Y. Wang, Z. Chen, Z. Wu, X. Ye, and X. Xu, "Pilates for overweight or obesity: a meta-analysis," Frontiers in Physiology, vol. 12, p. 643455, 2021.

[18] T. Van Criekinge, S. Truijen, J. Schröder et al., "The effectiveness of trunk training on trunk control, sitting and standing balance and mobility post-stroke: a systematic review and meta-analysis," Clinical Rehabilitation, vol. 33, no. 6, pp. 992-1002, 2019.

[19] Z. Chen, X. Ye, Y. Xia et al., "Effect of pilates on glucose and lipids: a systematic review and meta-analysis of randomized controlled trials," Frontiers in Physiology, vol. 12, Article ID 641968, 2021.

[20] G. H. Guyatt, A. D. Oxman, G. E. Vist et al., "GRADE: an emerging consensus on rating quality of evidence and strength of recommendations," BMJ, vol. 336, no. 7650, pp. 924-926, 2008.

[21] S. Li, M. Zheng, W. Wu, J. Guo, F. Ji, and Z. Zheng, "Effects of electroacupuncture administered 24hours prior to surgery on postoperative nausea and vomiting and pain in patients undergoing gynecologic laparoscopic surgery: a feasibility study," Explore, vol. 13, no. 5, pp. 313-318, 2017.

[22] S. Praveena Seevaunnamtum, K. Bhojwani, and N. Abdullah, "Intraoperative electroacupuncture reduces postoperative pain, analgesic requirement and prevents postoperative nausea and vomiting in gynaecological surgery: a randomised controlled trial," Anesthesiology and Pain Medicine, vol. 6, no. 6, Article ID e40106, 2016.

[23] H. Bai, J. Sun, and J. Zhang, "Effect of electroacupuncture combined with tropisetron in treating postoperative nausea and vomiting in patients undergoing total hysterectomy," Journal of Clinical Anesthesiology, vol. 28, no. 12, pp. 1158-1160, 2012.

[24] W. Chen, G. Fu, L. Wang, L. Yuan, W. Shen, and M. Zhang, "The effect of electroacupuncture at tsusanli on gastric mucosal blood gas in gynecological laparoscopic surgery," Journal of Clinical Anesthesiology, vol. 30, no. 08, pp. 781-784, 2014.

[25] C. Jin and X. Jing, "Effect of electroacupuncture at Zusanli on recovery of gastrointestinal function and electrolyte level after total hysterectomy," Journal of new Chinese medicine, vol. 47, no. 05, pp. 248-249, 2015.

[26] S. Li, G. Yang, X. Wang, M. Zheng, and S. Ji, "Effect of preoperative electroacupuncture at different times on the quality of early postoperative recovery in patients undergoing total abdominal hysterectomy," Jiangsu Journal of Traditional Chinese Medicine, vol. 51, no. 12, pp. 65-67, 2019.

[27] Y. Lu, W. Tian, G. Xu et al., "Effect of electroacupuncture on morphine analgesia inducing digestive function disorder in patients undergoing hysterectomy," Practical Pharmacy and Clinical Remedies, vol. 13, no. 6, pp. 405-406, 2010.

[28] Toronui-Turson, "Effect of electroacupuncture zusanli on laparoscopic surgery in gynecological Patients," China Health Standard Management, vol. 10, no. 23, pp. 94-97, 2019.

[29] X. Wang, W. Wu, M. Zheng, and S. Li, "Randomized controlled pilot trial of electroacupuncture for prevention and treatment of gastrointestinal dysfunction after operation,"
Journal of Liaoning university of TCM, vol. 20, no. 06, pp. 179-182, 2018.

[30] X. Wang, W. Wu, M. Zheng, and S. Li, "Preoperative electroacupuncture on gastrointestinal function in patients with gynecologic laparoscopy," Journal of Clinical Acupuncture and Moxibustion, vol. 34, no. 02, pp. 35-38, 2018.

[31] X. Wang, Linqin, X. Yang et al., "The effect of Electroacupuncture on gastrointestinal complications after laparoscopic surgery," Fujian journal of TCM, vol. 42, no. 6, pp. 9-10, 2011.

[32] Y. Wang and Y. Xi, "Treatment of 40 cases of gastrointestinal dysfunction after gynecological abdominal operation by electroacupuncture combined with umbilical moxibustion," Henan traditional Chinese medicine, vol. 37, no. 03, pp. 538-540, 2017.

[33] Y. Xi and Y. Wang, "Therapeutic observation of electroacupuncture plus umbilical moxibustion for gastrointestinal dysfunction after gynecological abdominal operation," Shanghai Journal of Acupuncture and Moxibustion, vol. 34, no. 11, pp. 1076-1079, 2015.

[34] Q. Yang, W. Ma, and Y. Li, "Comparison of the effects of acupuncture-assisted anesthesia with different acupoints combination in gynecological laparoscopic operation," Chinese Acupuncture and Moxibustion, vol. 32, no. 1, pp. 59-64, 2012.

[35] C. Huang, Z. Dai, X. Jin, and Y. Chen, "Effect of preoperative acupuncture at bilateral neiguan points on postoperative nausea and vomiting andplasma gastrointestinal hormone levels after gynecological laparoscopy," Journal of Shenyang Medical College, vol. 23, no. 02, pp. 129-131+166, 2021.

[36] C. Ye and F. Huang, "Effect of electroacupuncture at Neiguan on nausea and vomiting after gynecological laparoscopic surgery," World Latest Medicine Information, vol. 19, no. 93, pp. 223-224, 2019.

[37] Y. Yu and C. Ning, "Effects of electroacupuncture on preventing postoperative nausea and vomiting after gynecological laparoscopic surgery," Chinese Journal of Woman and Child Health Research, vol. 27, no. 08, pp. 1015-1017, 2016.

[38] He Zhang, Wanglan, M. Zhang et al., "Electroacupuncture for improving the $\mathrm{CO} 2$ pneumoperitoneum stress injury during surgery," Journal of Clinical Acupuncture and Moxibustion, no. 8, pp. 30-35, 2013.

[39] A. Lee, S. K. Chan, and L. T. Fan, "Stimulation of the wrist acupuncture point PC6 for preventing postoperative nausea and vomiting," Cochrane Database of Systematic Reviews, vol. 2015, no. 11, Article ID CD003281, 2015.

[40] C. Y. Chen and C. Y. Tsai, "Ghrelin and motilin in the gastrointestinal system," Current Pharmaceutical Design, vol. 18, no. 31, pp. 4755-4765, 2012.

[41] Y. Liu, L. Yang, and S. J. Tao, "Effects of hydromorphone and morphine intravenous analgesia on plasma motilin and postoperative nausea and vomiting in patients undergoing total hysterectomy," European Review for Medical and Pharmacological Sciences, vol. 22, no. 17, pp. 5697-5703, 2018.

[42] J. Pascual-Ramírez, L. G. Collar Viñuelas, J. Martín, G. Bernal, A. Bosque Castro, and N. García-Serrano, "Mucosal tonometry as early warning of gastrojejunal leak in laparoscopic Roux-en-y gastric bypass," Obesity Surgery, vol. 22, no. 5, pp. 843-846, 2012.

[43] N. Arya, M. A. Sharif, L. L. Lau et al., "Retroperitoneal approach to abdominal aortic aneurysm repair preserves splanchnic perfusion as measured by gastric tonometry," Annals of Vascular Surgery, vol. 24, no. 3, pp. 321-327, 2010. 
[44] Y. Hayakawa, W. Chang, G. Jin, and T. C. Wang, "Gastrin and upper GI cancers," Current Opinion in Pharmacology, vol. 31, pp. 31-37, 2016.

[45] M. Iwasaki, Y. Akiba, and J. D. Kaunitz, "Recent advances in vasoactive intestinal peptide physiology and pathophysiology: focus on the gastrointestinal system," 2019.

[46] J. J. Cullen, J. C. Eagon, and K. A. Kelly, "Gastrointestinal peptide hormones during postoperative ileus. Effect of octreotide," Digestive Diseases and Sciences, vol. 39, no. 6, pp. 1179-1184, 1994.

[47] M. Okada, K. Itoh, H. Kitakoji, and K. Imai, "Mechanism of electroacupuncture on postoperative ileus induced by surgical stress in rats," Medical Acupuncture, vol. 31, no. 2, pp. 109-115, 2019.

[48] N. N. Yang, Y. Ye, Z. X. Tian et al., "Effects of electroacupuncture on the intestinal motility and local inflammation are modulated by acupoint selection and stimulation frequency in postoperative ileus mice," Neuro-Gastroenterology and Motility, vol. 32, no. 5, Article ID e13808, 2020.

[49] K. F. Schulz, D. G. Altman, D. Moher et al., "CONSORT 2010 statement: updated guidelines for reporting parallel group randomised trials," Trials, vol. 11, no. 1, p. 32, 2010. 